\title{
Design, Synthesis and in vitro Anti-Zika virus evaluation of novel Sinefungin derivatives
}

Zeyu Tao, ${ }^{\mathrm{a}, 1}$ Ruiyuan Cao, ${ }^{\mathrm{b}, 1}$ Yunzheng Yan, ${ }^{\mathrm{b}}$ Guocheng Huang, ${ }^{\mathrm{a}} \mathrm{Kai} \mathrm{Lv},{ }^{\mathrm{a},{ }^{*}}$ Wei Li, ${ }^{\mathrm{b}}$ Yunhe Geng, ${ }^{a}$ Lei Zhao, ${ }^{\mathrm{b}}$ Apeng Wang, ${ }^{\mathrm{a}}$ Qinghao He, ${ }^{\mathrm{b}}$ Jingjing Yang, ${ }^{\mathrm{b}}$ Shiyong Fan, ${ }^{\mathrm{b}}$ Menghao Huang, Huiyuan Guo, ${ }^{\mathrm{a}}$ Wu Zhong, ${ }^{\mathrm{b}}$ Mingliang Liu,

${ }^{a}$ Institute of Medicinal Biotechnology, Chinese Academy of Medical Sciences and Peking Union Medical College, Beijing 100050, China

${ }^{b}$ National Engineering Research Center for the Emergence Drugs, Beijing Institute of Pharmacology and Toxicology, Beijing, 100850, China

${ }^{\mathrm{c}}$ Division of Gastroenterology and Hepatology, Department of Medicine, Indiana University School of Medicine, Indiana 46202, USA

${ }^{1}$ These authors contributed equally to this work.

*Corresponding authors: 1vkailk@ hotmail.com, 86-010-63165280 (K. Lv); 1mllyx@126.com, 86010-63030965 (M.L. Liu).

KEYWORDS: anti-Zika virus, Sinefungin, EPZ004777, structure-activity relationships, methyltransferases

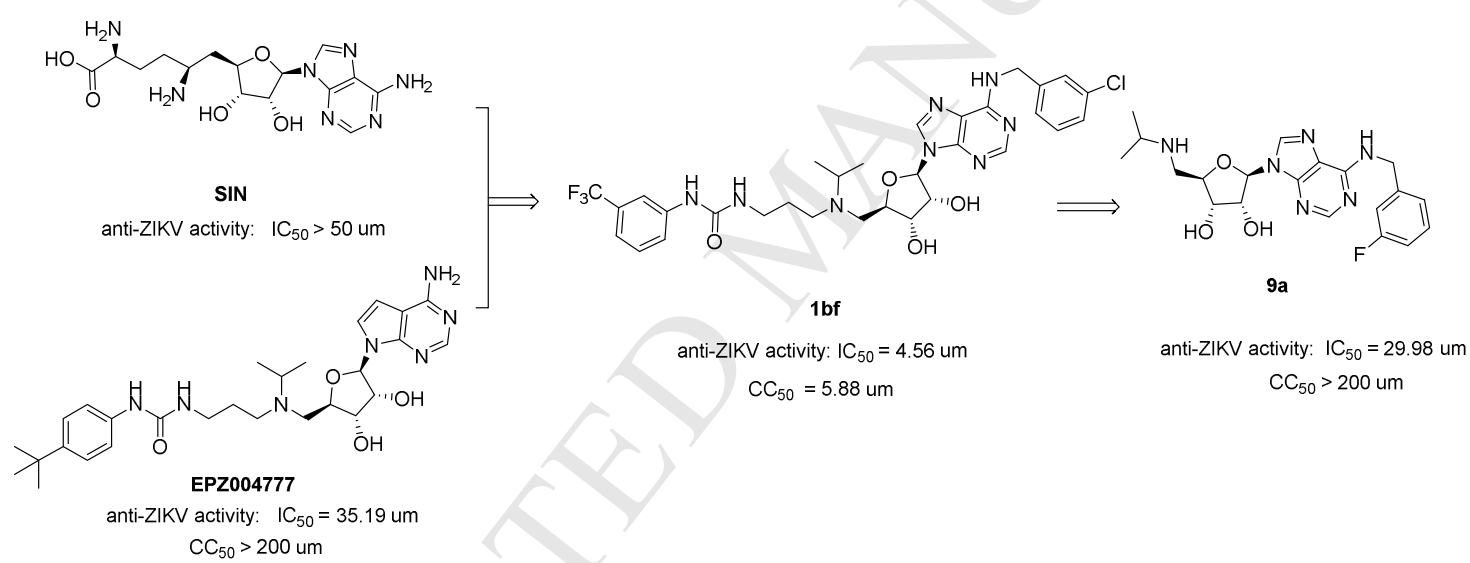

ABSTRACT: 1 bf shows better activity $\left(\mathrm{IC}_{50}=4.56 \mu \mathrm{M}\right)$ than EPZ004777 $\left(\mathrm{IC}_{50}=35.19 \mu \mathrm{M}\right)$. Intermediate 9a displays good activity $\left(\mathrm{IC}_{50}=29.98 \mu \mathrm{M}\right)$ and acceptable cytotoxicity $\left(\mathrm{CC}_{50}>200\right.$ $\mu \mathrm{M})$.

This is the author's manuscript of the article published in final edited form as:

Tao, Z., Cao, R., Yan, Y., Huang, G., Lv, K., Li, W., ... \& Yang, J. (2018). Design, synthesis and in vitro antiZika virus evaluation of novel Sinefungin derivatives. European journal of medicinal chemistry, 157, 994-1004. https://doi.org/10.1016/j.ejmech.2018.08.057 


\section{Design, Synthesis and in vitro Anti-Zika virus evaluation of novel Sinefungin derivatives}

Zeyu Tao, ${ }^{\mathrm{a}, 1}$ Ruiyuan Cao, ${ }^{\mathrm{b}, 1}$ Yunzheng Yan, ${ }^{\mathrm{b}}$ Guocheng Huang, ${ }^{\mathrm{a}} \mathrm{Kai} \mathrm{Lv},{ }^{\mathrm{a},{ }^{*}}$ Wei Li, ${ }^{\mathrm{b}}$ Yunhe Geng, ${ }^{\mathrm{a}}$ Lei Zhao, ${ }^{\mathrm{b}}$ Apeng Wang, ${ }^{\mathrm{a}}$ Qinghao He, ${ }^{\mathrm{b}}$ Jingjing Yang, ${ }^{\mathrm{b}}$ Shiyong Fan, ${ }^{\mathrm{b}}$ Menghao Huang, ${ }^{\mathrm{c}}$ Huiyuan Guo, ${ }^{\mathrm{a}}$ Wu Zhong, ${ }^{\mathrm{b}}$ Mingliang Liu,

a Institute of Medicinal Biotechnology, Chinese Academy of Medical Sciences and Peking Union Medical College, Beijing 100050, China

${ }^{\mathrm{b}}$ National Engineering Research Center for the Emergence Drugs, Beijing Institute of Pharmacology and Toxicology, Beijing, 100850, China

${ }^{c}$ Division of Gastroenterology and Hepatology, Department of Medicine, Indiana University School of Medicine, Indiana 46202, USA

${ }^{1}$ These authors contributed equally to this work.

*Corresponding authors: 1vkailk@hotmail.com, 86-010-63165280 (K. Lv); 1mllyx@126.com, 86-010-63030965 (M.L. Liu).

KEYWORDS: anti-Zika virus, Sinefungin, EPZ004777, structure-activity relationships, methyltransferases

ABSTRACT: We report herein the design and synthesis of a series of novel Sinefungin (SIN) derivatives, based on the structures of SIN and its analogue EPZ004777. Our results reveal that target compounds 1ad-af, 1ba-bb and $\mathbf{1 b f}$-bh show better activity $\left(\mathrm{IC}_{50}=4.56-20.16 \mu \mathrm{M}\right)$ than EPZ004777 $\left(\mathrm{IC}_{50}=35.19 \mu \mathrm{M}\right)$. Surprisingly, SIN was founded to be not as active $\left(\mathrm{IC}_{50}>50 \mu \mathrm{M}\right)$ as we and other research groups predicted. Interestingly, the intermediates $\mathbf{9 a - b}$ and $\mathbf{1 1 b}$ display potent anti-ZIKV potency $\left(\mathrm{IC}_{50}=6.33-29.98 \mu \mathrm{M}\right)$, and compound 9a also exhibits acceptable cytotoxicity $\left(\mathrm{CC}_{50}>200 \mu \mathrm{M}\right)$, suggesting their promising potential to be leads for further development.

\section{Introduction}

Zika virus (ZIKV) was first identified in 1947 in the Zika forest of Uganda, where it was isolated from the blood of sentinel rhesus macaques. [1] It belongs to the flavivirus genus of the Flaviviridae family, is related to yellow fever virus (YFV), dengue virus (DENV) and west nile virus (WNV). In the decades following its discovery, ZIKV posed little concern to the general public as it remained relatively dormant. The first outbreak occurred on the island of Yap in the federated states of Microesia in April 2007. [2-3] Subsequently, ZIKV strains have become more prevalent, leading to an increase in global epidemics. More than 60 countries and territories had reported ZIKV infection since the first epidemic. [4] Importantly, the ZIKV can lead to the rare birth defect microcephaly and other neurological disorders in infants and adults. [5] It was reported that over 3500 babies were born with microcephaly between Oct. 2015 and Jan. 2016 in Brazil. The world health organization (WHO) declared in November 2016 that the ZIKV is a highly significant and a long-term problem. [6] 


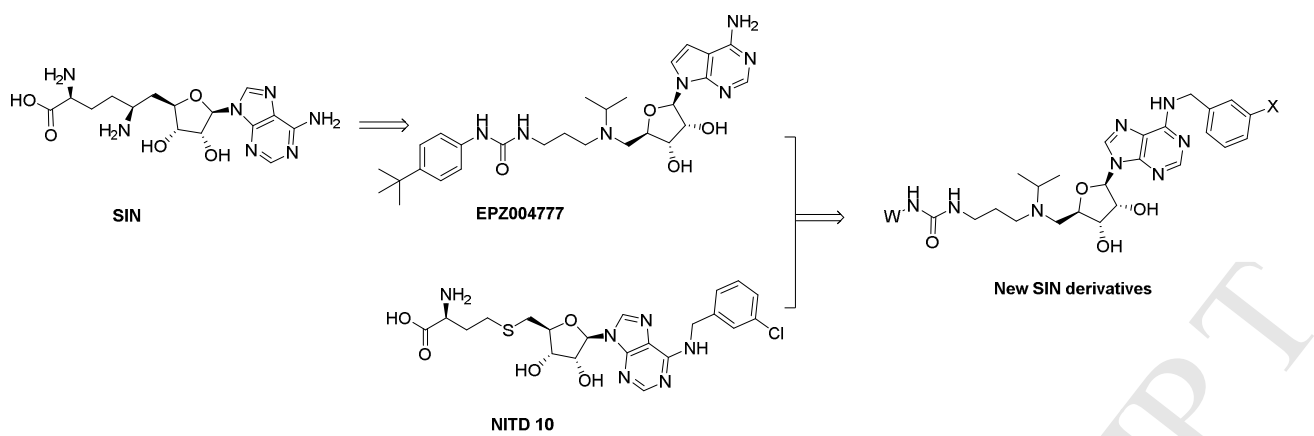

Figure 1. Design of new SIN derivatives

To date, there are no clinically approved vaccines or antiviral drugs for the treatment of ZIKV infection. Sinefungin (SIN, Figure 1) was isolated from the fermentation broth of Streptomyces griseoleus NRRL 3739 by Eli Lilly as a potential antifungal antibiotic. [7-8] It is structurally similar to S-Adenosyl methionine (SAM) and acts as a competitive inhibitor of numerous Methyltransferases (Mtases). It was reported that SIN exhibited antitumor, [9] antiviral [10-11] and antiparasitic activity [12]. Recently, SIN was demonstrated to show considerable anti-flavivirus activity (WNV, DENV-2 and YFV). Since ZIKV belongs to the flavivirus family, we agreed with others [13-15] in speculating that SIN might also be a potential ZIKV inhibitor.
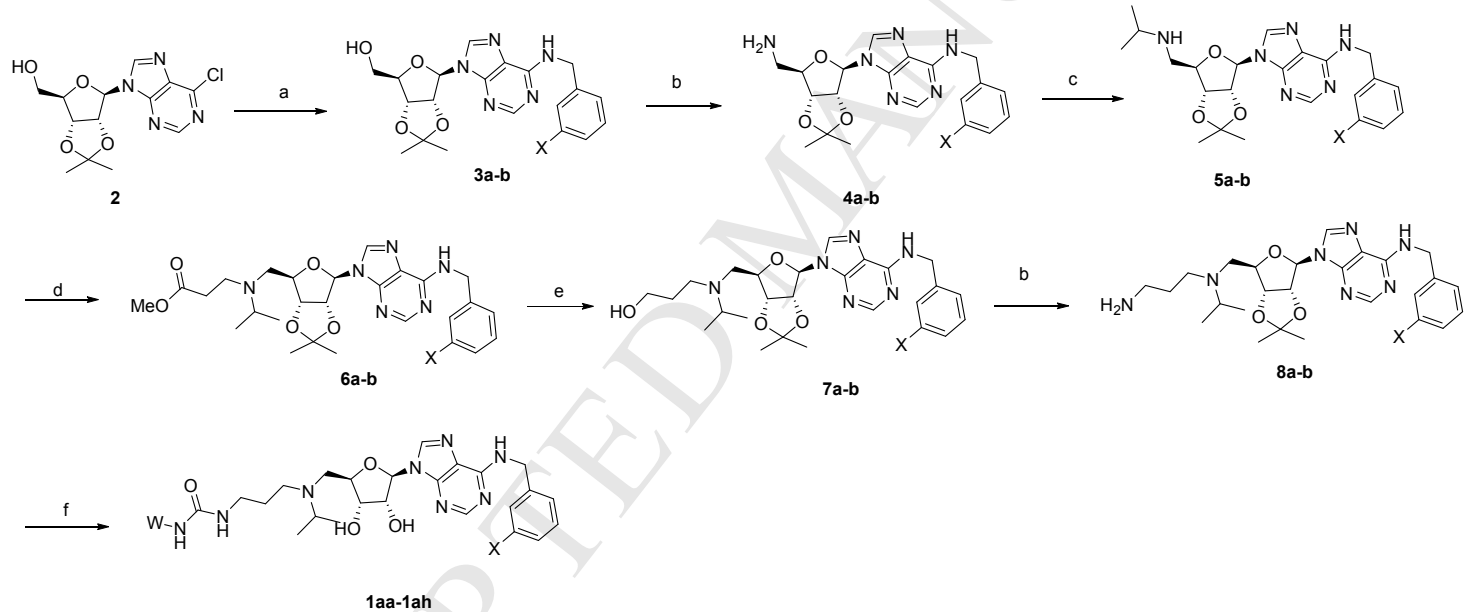

$$
\begin{aligned}
& \text { 1aa-1ah } \\
& \text { 1ba-1bh }
\end{aligned}
$$

Reagents and conditions: a) amines, $\mathrm{Et}_{3} \mathrm{~N}$, EtOH, reflux; b) i: DIAD, phthalimide, $\mathrm{Ph}{ }_{3}^{\mathrm{P}}, \mathrm{THF}, \mathrm{rt}$; ii: $85 \% \mathrm{NH}_{2} \mathrm{NH}_{2} . \mathrm{H}_{2} \mathrm{O}, \mathrm{EtOH}$, reflux; c) $\mathrm{NaCNBH}$, acetone, $\mathrm{AcOH}$, MeOH, rt; d) Methyl acrylate, MeOH, microwave, $80^{\circ} \mathrm{C}$; e) $\mathrm{LiAlH}_{4}, \mathrm{THF}, 0^{\circ} \mathrm{C}$; f) i: isocyanate, DCM; ii: TFA, DCM.

\section{Scheme 1. Synthesis of the target compounds}

SIN is water soluble, and displays a lower $\log \mathrm{P}$ value of -3.01 (calculated by chemdraw 16.0) which limits its membrane permeability. [13, 16] EPZ004777, a SIN structural analogue containing a lipophilicity side chain, was developed as a potent histone methyltransferase inhibitor. [17] We hypothesized that EPZ004777 could address the issue of SIN membrane permeability while retaining the anti-ZIKV activity.

Recently, the crystal structural of ZIKV Mtase with SIN was resolved by Kamil Hercik et al. Based on the structural data, they suggested modification of the adenine moiety of SIN to increase selectivity and binding affinity of ZIKV Mtase. [14] Additionally, researchers from Novartis Institute for Tropical Disease (NITD) discovered NITD 10, a $S$-adenosyl-1-homocysteine (SAH) analogue with 3-chloro-benzyl at the N-6 position of adenine. It selectively inhibited DENV-3 Mtase $(\mathrm{Ki}=0.002-0.24 \mu \mathrm{M})$ and WNV Mtase $(\mathrm{Ki}=0.044-5.68$ $\mu \mathrm{M})$ without suppressing host MTases $(\mathrm{Ki}>50 \mu \mathrm{M})$. [18] Thus, we sought to design, synthesis 
and evaluate a new series of SIN derivatives containing 3-fluoro- or chloro-benzyl moieties at $\mathrm{N}-6$ position of adenine and a lipophilicity side chain (aryl W) while integrating the structure features of NITD 10 and EPZ004777.

\section{Results and discussion}

\subsection{Chemistry}

The synthesis of new SIN derivatives 1 is shown in scheme 1. Coupling of the chloropurine 2 with substituted benzyl amines in EtOH at reflux resulted in adenines 3a-b. The primary alcohol of 3a-b was converted to the corresponding amines 4a-b via standard Mitsunobu displacement with phthalimide followed by hydrazinolysis in $75-77 \%$ yields over two steps. Reductive amination of $\mathbf{4 a}-\mathbf{b}$ with acetone in the presence of $\mathrm{NaCNBH}_{3}$ and acetic acid provided isopropyl amines 5a-b in good yields. Michael addition of compounds 5a-b with methyl acrylate in $\mathrm{MeOH}$ at reflux over 24 hours furnished 6a-b in a low yield, but under microwave radiation at $90{ }^{\circ} \mathrm{C}$ over 3 hours gave $\mathbf{6 a - b}$ in $76-80 \%$ yields. Reduction of methyl esters $\mathbf{6 a - b}$ with $\mathrm{LiAlH}_{4}$ in THF afforded alcohols $\mathbf{7 a - b}$, which upon Mitsunobu reaction and hydrazinolysis generated amines 8a-b. Treatment of 8a-b with isocyanate in DCM followed by removal of the acetonide protecting group with TFA furnished the SIN derivatives 1ab-af, and $\mathbf{1 b a - b f}$.

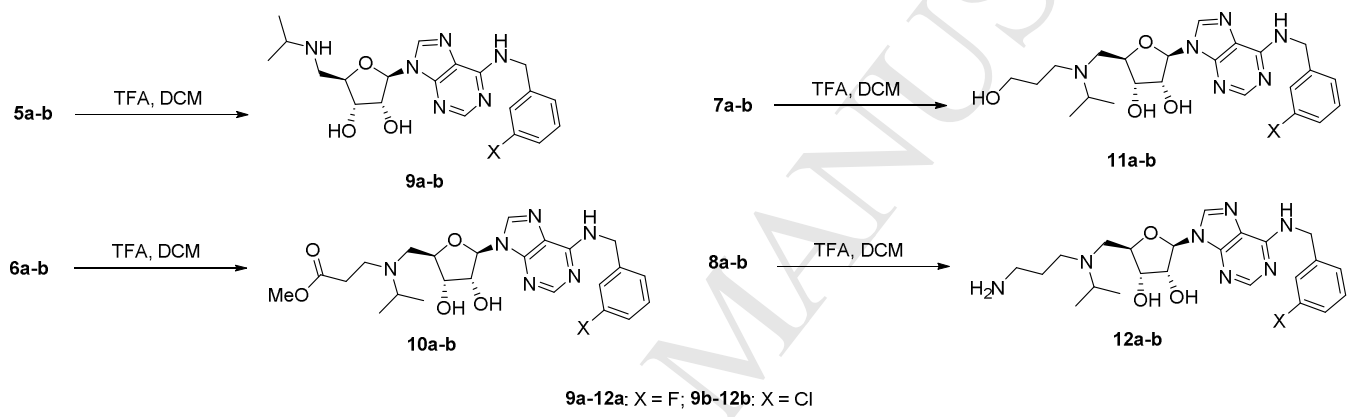

Scheme 2. Synthesis of compounds 9-12

To achieve structurally diverse targets, the acetonide protecting group of the intermediates 5-8 was also removed by TFA in DCM, as outlined in scheme 2. The resulting compounds 9-12 were also tested for their anti-ZIKV activity.

\subsection{Anti-ZIKV activity}

All new SIN derivatives 1aa-ah, $\mathbf{1 b a}-\mathbf{b h}$, and deprotected intermediates 9-12 were evaluated for their in vitro anti-ZIKV activity in an infection-based cell culture model that utilizes a ZIKV strain SMGC and BHK cell line by Cell Titer-Glo Luminescent Cell Viability Assay (Promega). The ZIKV was obtained from the Institute of Microbiology, Chinese Academy of Sciences, Beijing, China. The $\mathrm{IC}_{50}$ values of the SIN derivatives 1aa-ah, 1ba-bh and intermediates 9-12 along with SIN and EPZ004777 for comparison were summarized in $\mu \mathrm{M}$ in Tables 1 and 2, respectively.

Surprisingly, $\mathrm{SIN}\left(\mathrm{IC}_{50}>50 \mu \mathrm{M}\right)$ is not as active against the ZIKV as we predicted, whereas EPZ004777 displays considerable anti-ZIKV activity $\left(\mathrm{IC}_{50}=35.19 \mu \mathrm{M}\right)$. Among the target compounds, 1ad-af, 1ba-bb, and 1bf-bh exhibit potent activity $\left(\mathrm{IC}_{50}<30 \mu \mathrm{M}\right) ; \mathbf{1 a a - a c}, \mathbf{1 a g}-\mathbf{a h}$, and $\mathbf{1 b c}$ are not active against ZIKV; 1bd-be are failure to acquire anti-ZIKV activity in this model, possibly due to their high toxicity $\left(\mathrm{CC}_{50}=6.35-6.73 \mu \mathrm{M}\right)$ in BHK cell line. In particular, compounds 1ad-af, $\mathbf{1 b b}$, and $\mathbf{1 b f}\left(\mathrm{IC}_{50}=4.56-6.99 \mu \mathrm{M}\right)$ were found to be 5.0-7.7-fold more potent than the reference EPZ004777.

Generally, compounds with chlorine as the aryl X substituent display higher activity than the corresponding fluorine analogues (1aa-ab vs 1ba-bb; 1af-ah vs 1bf-bh). Notably, this SAR result is consistent with an earlier report on the DENV-related SAR observations by NITD, [18] suggesting that the Mtase structures of ZIKV and DENV might be similar. When the aryl X 
substituent is fluorine, compounds 1ad-af with 4- $t$-Bu-phenyl, 4- $\mathrm{CF}_{3}$-phenyl and 3- $\mathrm{CF}_{3}$-phenyl as the aryl $\mathrm{W}$ group show excellent anti-ZIKV potency $\left(\mathrm{IC}_{50}=6.46-6.99 \mu \mathrm{M}\right)$. In contrast, introduction of other aryl W substituents leads to a compete loss of activity (1aa-ac and 1ag-ah, $\left.\mathrm{IC}_{50}>50 \mu \mathrm{M}\right)$. When the aryl X substituent is chlorine, apart from a few exceptions (1bc-be), all of these compounds display potent activity $\left(\mathrm{IC}_{50}<30 \mu \mathrm{M}\right.$ ), and the contribution of aryl $\mathrm{W}$ group to the activity is in this order: $3-\mathrm{CF}_{3}$-phenyl $>4-\mathrm{Me}$-phenyl $>$ cyclohexyl $>4-\mathrm{F}$-phenyl $>t$-Bu $>$ 4-methoxyphenyl.

Subsequently, intermediates 9-12 were tested for their anti-ZIKV activity (Table 2). Most of the intermediates 10a-b, 11a and 12a-b are not active $\left(\mathrm{IC}_{50}>50 \mu \mathrm{M}\right)$ but $9 \mathbf{a}, 9 \mathbf{b}$ and $11 \mathbf{b}\left(\mathrm{IC}_{50}=\right.$ 6.33-29.98 $\mu \mathrm{M})$ fortunately display potent anti-ZIKV activity. Among them, compound $\mathbf{9 b}\left(\mathrm{IC}_{50}\right.$ $=6.33 \mu \mathrm{M}$ ) demonstrated to be more potent than its fluorne analogue 10a. Interestingly, this SAR is similar as above in the SIN derivatives.

Table 1. Structures and anti-ZIKV activity of the target compounds

\begin{tabular}{|c|c|c|c|c|c|}
\hline Compds. & $\mathrm{X}$ & $\mathrm{W}$ & $\mathrm{IC}_{50}(\mu \mathrm{M})$ & $\mathrm{CC}_{50}(\mu \mathrm{M})$ & SI \\
\hline 1aa & $\mathrm{F}$ & 4-F-phenyl & $>50$ & ND & NA \\
\hline 1ab & $\mathrm{F}$ & 4-Me-phenyl & $>50$ & ND & NA \\
\hline $1 \mathrm{ac}$ & $\mathrm{F}$ & 4-MeO-phenyl & $>50$ & ND & NA \\
\hline 1ad & $\mathrm{F}$ & 4-t-Bu-phenyl & $6.65 \pm 0.07$ & $18.71 \pm 0.76$ & 2.81 \\
\hline 1ae & $\mathrm{F}$ & 4- $\mathrm{CF}_{3}$-phenyl & $6.46 \pm 0.22$ & $20.27 \pm 0.93$ & 3.14 \\
\hline 1af & F & 3- $\mathrm{CF}_{3}$-phenyl & $6.99 \pm 0.39$ & $12.88 \pm 0.28$ & 1.84 \\
\hline 1ag & $\mathrm{F}$ & $t-\mathrm{Bu}$ & $>50$ & ND & NA \\
\hline 1ah & $\mathrm{F}$ & Cyclohexyl & $>50$ & ND & NA \\
\hline $1 \mathrm{ba}$ & $\mathrm{Cl}$ & 4-F-phenyl & $15.29 \pm 8.37$ & $20.01 \pm 7.83$ & 1.31 \\
\hline $1 \mathrm{bb}$ & $\mathrm{Cl}$ & 4-Me-phenyl & $5.80 \pm 1.42$ & $19.11 \pm 0.76$ & 3.29 \\
\hline $1 \mathrm{bc}$ & $\mathrm{Cl}$ & 4-MeO-phenyl & $>50$ & ND & NA \\
\hline 1bd & $\mathrm{Cl}$ & 4- $t$-Bu-phenyl & NA & $6.73 \pm 0.02$ & NA \\
\hline 1be & $\mathrm{Cl}$ & 4- $\mathrm{CF}_{3}$-phenyl & NA & $6.35 \pm 1.13$ & NA \\
\hline $1 \mathrm{bf}$ & $\mathrm{Cl}$ & 3- $\mathrm{CF}_{3}$-phenyl & $4.56 \pm 3.84$ & $5.88 \pm 1.62$ & 1.29 \\
\hline $1 \mathrm{bg}$ & $\mathrm{Cl}$ & $t-\mathrm{Bu}$ & $20.61 \pm 0.38$ & $58.49 \pm 1.16$ & 2.84 \\
\hline 1bh & $\mathrm{Cl}$ & Cyclohexyl & $12.24 \pm 4.02$ & $57.57 \pm 1.42$ & 4.70 \\
\hline SIN & & & $>50$ & ND & NA \\
\hline EPZ004777 & & & $35.19 \pm 7.02$ & $>200$ & $>5.68$ \\
\hline
\end{tabular}

ND, not detected; NA, not available.

\subsection{Cytotoxicity}

The SIN derivatives and additional intermediates that displayed considerable anti-ZIKV activity $\left(\mathrm{IC}_{50}<50 \mu \mathrm{M}\right)$ were further evaluated for cytotoxicity test against BHK cell line by Cell Titer-Glo Luminescent Cell Viability Assay (Promega). Unfortunately, all the tested SIN derivatives $\left(\mathrm{CC}_{50}=5.88-58.49 \mu \mathrm{M}\right)$ exhibit higher cytotoxicity than EPZ004777 $\left(\mathrm{CC}_{50}>200\right.$ $\mu \mathrm{M})$, and their selective indexes $(\mathrm{SI}=1.29-4.70)$ are lower than EPZ004777 (SI > 5.68). The best compound $\mathbf{1 b f}$ displays the highest cytotoxicity $\left(\mathrm{CC}_{50}=5.88 \mu \mathrm{M}\right)$, the least $\mathbf{1 b g}$ also exhibits the lowest cytotoxicity $\left(\mathrm{CC}_{50}=58.49 \mu \mathrm{M}\right)$. Considering both of the activity and cytotoxicity, compound $\mathbf{1 b h}$ which holds the highest selective index $(\mathrm{SI}=4.70)$ among these analogues could be selected as a lead compound for further modification.

To our delight, we observed that compound 9a shows acceptable cytotoxicity $\left(\mathrm{CC}_{50}>200\right.$ $\mu \mathrm{M})$ and potent anti-ZIKV activity $\left(\mathrm{IC}_{50}=29.98 \mu \mathrm{M}\right)$, suggesting compound $9 \mathrm{a}$ might be a potent 
candidate for further development. Compounds $\mathbf{9 b}$ and $\mathbf{1 1 b}$ with higher cytotoxicity $\left(\mathrm{CC}_{50}=\right.$ 29.39-70.61 $\mu \mathrm{M})$ deserve further modification in the future.

\section{Conclusion}

In summary, the natural product SIN was selected as our lead compound but proved to be inactive in this study, whereas its structural analogue EPZ004777 displays considerable anti-ZIKV activity $\left(\mathrm{IC}_{50}=35.19 \mu \mathrm{M}\right)$. A series of structural unique SIN derivatives with a 3-fluoro or chloro-benzyl group and a lipophilicity side chain were designed, synthesized and evaluated for anti-ZIKV activity. SIN derivatives 1ad-af, 1ba-bb, and $\mathbf{1 b f}-\mathbf{b h}$ show more potent activity $\left(\mathrm{IC}_{50}=4.56-20.16 \mu \mathrm{M}\right)$ than EPZ004777, but their $\mathrm{CC}_{50}$ values are lower than EPZ004777 $\left(\mathrm{CC}_{50}>200 \mu \mathrm{M}\right)$. We also observed that intermediates 9a-b and $\mathbf{1 1 b}$ display potent anti-ZIKV activity $\left(\mathrm{IC}_{50}=6.33-29.98 \mu \mathrm{M}\right)$. These intermediates, possessing a simplified structure, could be selected as new lead compounds for further studies. In addition, we found that compound 9a displays acceptable cytotoxicity $\left(\mathrm{CC}_{50}>200 \mu \mathrm{M}\right)$, suggesting its promising potential as a candidate for further development. Studies to determine the in vivo efficacy of $\mathbf{9 a}$ are currently underway.

Table 2. Anti-ZIKV activity and cytotoxicity of compounds 9-12

\begin{tabular}{llll}
\hline Compds. & $\mathrm{IC}_{50}(\mu \mathrm{M})$ & $\mathrm{CC}_{50}(\mu \mathrm{M})$ & $\mathrm{SI}$ \\
\hline $\mathbf{9 a}$ & $29.98 \pm 6.93$ & $>200$ & $>6.67$ \\
$\mathbf{9 b}$ & $6.33 \pm 1.93$ & $29.39 \pm 8.55$ & 4.64 \\
$\mathbf{1 0 a}$ & $>50$ & $\mathrm{ND}$ & $\mathrm{NA}$ \\
$\mathbf{1 0 b}$ & $>50$ & $\mathrm{ND}$ & $\mathrm{NA}$ \\
$\mathbf{1 1 a}$ & $>50$ & $\mathrm{ND}$ & $\mathrm{NA}$ \\
$\mathbf{1 1 b}$ & $27.47 \pm 4.32$ & $70.61 \pm 0.05$ & 2.57 \\
$\mathbf{1 2 a}$ & $>50$ & $\mathrm{ND}$ & $\mathrm{NA}$ \\
$\mathbf{1 2 b}$ & $>50$ & $\mathrm{ND}$ & $\mathrm{NA}$ \\
SIN & $>50$ & $\mathrm{ND}$ & NA \\
EPZ004777 & 35.19 & $>200$ & $>5.68$ \\
\hline ND, not detected; NA, not available.
\end{tabular}

\section{Experimental protocols}

\subsection{Chemistry}

${ }^{1} \mathrm{H}$ NMR spectra were determined on a Varian Mercury-400 or Bruker $500 \mathrm{M}$ spectrometer in $\mathrm{MeOD}$ or $\mathrm{CDCl}_{3}$ using tetramethylsilane as an internal standard. Electrospray ionization (ESI) mass spectra was obtained on an Agilent 1260-6420 Mass spectrum instruments. The reagents were all of analytical grade or chemically pure. TLC was performed on silica gel plates (Merck, ART5554 60F254). All the anhydrous solvents were purchased from J\&K Scientific.

\subsection{Synthesis}

4.2.1. ((3aR,4R,6R,6aR)-6-(6-((3-fluorobenzyl)amino)-9H-purin-9-yl)-2,2-dimethyl tetrahydrofuro[3,4-d][1,3]dioxol-4-yl)methanol 3a

To a stirred solution of compound $2(3 \mathrm{~g}, 9.2 \mathrm{mmol})$ in $\mathrm{EtOH}(50 \mathrm{~mL})$ was added $\mathrm{Et}_{3} \mathrm{~N}(2.6$ $\mathrm{mL}, 18.4 \mathrm{mmol})$ and 3-fluorobenzylamine $(2.1 \mathrm{~mL}, 18.4 \mathrm{mmol})$ at room temperature. The mixture was stirred for 5 hours at $40{ }^{\circ} \mathrm{C}$ and concentrated. The residue was purified by column chromatography over silica gel (DCM : $\left.\mathrm{MeOH}: \mathrm{NH}_{3} \mathrm{H}_{2} \mathrm{O}=200: 10: 0.1\right)$ to yield compound 3a $(3.13 \mathrm{~g}, 82 \%)$ as a colorless oil; $[\alpha]^{20}=-167.77(\mathrm{c} 0.78, \mathrm{MeOH}) ;{ }^{1} \mathrm{H}$ NMR $\left(500 \mathrm{MHz}, \mathrm{CDCl}_{3}\right) \delta$ $8.39(\mathrm{~s}, 1 \mathrm{H}$, purin- $\mathrm{H}), 7.79(\mathrm{~s}, 1 \mathrm{H}$, purin- $\mathrm{H}), 7.35-7.30(\mathrm{~m}, 1 \mathrm{H}, \mathrm{Ar}-\mathrm{H}), 7.17(\mathrm{~d}, \mathrm{~J}=7.5 \mathrm{~Hz}, 1 \mathrm{H}$, Ar-H), $7.11(\mathrm{~d}, \mathrm{~J}=9 \mathrm{~Hz}, 1 \mathrm{H}, \mathrm{Ar}-\mathrm{H}), 6.59$ (brs, 1H, NH), $5.89(\mathrm{~s}, 1 \mathrm{H}$, tetrahydrofuro-H), $5.24(\mathrm{t}, \mathrm{J}$ $=5.1 \mathrm{~Hz}, 1 \mathrm{H}$, tetrahydrofuro- $\mathrm{H}), 5.14(\mathrm{~d}, \mathrm{~J}=5.5 \mathrm{~Hz}, 1 \mathrm{H}$, tetrahydrofuro- $\mathrm{H}), 4.89$ (brs, $2 \mathrm{H}$, benzyl- $\left.\mathrm{CH}_{2}\right), 4.58(\mathrm{~s}, 1 \mathrm{H}$, tetrahydrofuro- $\mathrm{H}), 4.00\left(\mathrm{~d}, \mathrm{~J}=12.6 \mathrm{~Hz}, 1 \mathrm{H}, \mathrm{CH}_{2} \mathrm{OH}-\mathrm{CH}\right), 3.84(\mathrm{~d}, \mathrm{~J}=$ $\left.12.6 \mathrm{~Hz}, 1 \mathrm{H}, \mathrm{CH}_{2} \mathrm{OH}-\mathrm{CH}\right), 1.68\left(\mathrm{~s}, 3 \mathrm{H}, \mathrm{CH}_{3}\right), 1.42\left(\mathrm{~s}, 3 \mathrm{H}, \mathrm{CH}_{3}\right) ;{ }^{13} \mathrm{C} \mathrm{NMR}\left(400 \mathrm{MHz}, \mathrm{CDCl}_{3}\right) \delta$ 
$162.5(\mathrm{~d}, \mathrm{~J}=242.6 \mathrm{~Hz}, \mathrm{Ar}-\mathrm{C}$ ), 154.8 (purin-C), 152.8 (purin-C), 147.5 (purin-C), 140.7 (purin-C), 139.8 (Ar-C), $130.2(\mathrm{~d}, \mathrm{~J}=8.1 \mathrm{~Hz}$, Ar-C), $123.12(\mathrm{~d}, \mathrm{~J}=2.5 \mathrm{~Hz}, \mathrm{Ar}-\mathrm{H}), 121.2$ (purin-C), $114.5(\mathrm{~d}, \mathrm{~J}=21.8 \mathrm{~Hz}$, Ar-C), 114.0 (Ar-C), 94.4 (tetrahydrofuro-C), 86.1 (tetrahydrofuro-C), 83.1 (tetrahydrofuro-C), 81.7 (tetrahydrofuro-C), $63.4 \quad\left(\mathrm{CH}_{2}-\mathrm{C}\right), 43.8$ (benzyl- $\mathrm{CH}_{2}-\mathrm{C}$ ), $27.6\left(\mathrm{CH}_{3}\right), 25.2\left(\mathrm{CH}_{3}\right)$; LRMS (ESI): $\mathrm{m} / \mathrm{z}=438[\mathrm{M}+\mathrm{Na}]^{+}$.

4.2.2. ((3aR,4R,6R,6aR)-6-(6-((3-chlorobenzyl)amino)-9H-purin-9-yl)-2,2-dimethyl tetrahydrofuro[3,4-d][1,3]dioxol-4-yl)methanol 3b

Following above synthetic procedure of compound 3a, replacing 3-fluorobenzylamine with 3-chlorobenzyamine afforded compound $\mathbf{3 b}(85 \%)$ as a colorless oil, $[\alpha]^{20}{ }_{\mathrm{D}}=-59.03$ (c 0.93 , $\mathrm{MeOH}) ;{ }^{1} \mathrm{H}$ NMR $\left(500 \mathrm{MHz}, \mathrm{CDCl}_{3}\right) \delta 8.40(\mathrm{~s}, 1 \mathrm{H}$, purin-H), $7.79(\mathrm{~s}, 1 \mathrm{H}$, purin- $\mathrm{H}), 7.39(\mathrm{~s}, 1 \mathrm{H}$, Ar-H), 7.30-7.28 (m, 3H, Ar-H), 6.59 (brs, $1 \mathrm{H}, \mathrm{NH}), 5.89(\mathrm{~s}, 1 \mathrm{H}$, tetrahydrofuro- $\mathrm{H}), 5.24(\mathrm{t}, \mathrm{J}=$ $5.1 \mathrm{~Hz}, 1 \mathrm{H}$, tetrahydrofuro- $\mathrm{H}), 5.14(\mathrm{~d}, \mathrm{~J}=5.5 \mathrm{~Hz}, 1 \mathrm{H}$, tetrahydrofuro- $\mathrm{H}), 4.89$ (brs, $2 \mathrm{H}$, benzyl- $\left.\mathrm{CH}_{2}\right), 4.58(\mathrm{~s}, 1 \mathrm{H}), 4.00\left(\mathrm{~d}, \mathrm{~J}=12.6 \mathrm{~Hz}, 1 \mathrm{H}, \mathrm{CH}_{2} \mathrm{OH}-\mathrm{CH}\right), 3.84(\mathrm{~d}, \mathrm{~J}=12.6 \mathrm{~Hz}, 1 \mathrm{H}$, $\left.\mathrm{CH}_{2} \mathrm{OH}-\mathrm{CH}\right), 1.68\left(\mathrm{~s}, 3 \mathrm{H}, \mathrm{CH}_{3}\right), 1.42\left(\mathrm{~s}, 3 \mathrm{H}, \mathrm{CH}_{3}\right) ; \mathrm{LRMS}(\mathrm{ESI}): \mathrm{m} / \mathrm{z}=454[\mathrm{M}+\mathrm{Na}]^{+}$.

4.2.3.

9-((3aR,4R,6R,6aR)-6-(aminomethyl)-2,2-dimethyltetrahydrofuro[3,4-d][1,3]dioxol-4-yl)-N-(3-fl uorobenzyl)-9H-purin-6-amine $4 \mathbf{a}$

To a stirred solution of compound $3 \mathbf{a}(1 \mathrm{~g}, 2.4 \mathrm{mmol})$ in THF $(20 \mathrm{~mL})$ was added $\mathrm{Ph}_{3} \mathrm{P}(1.26$ $\mathrm{g}, 4.8 \mathrm{mmol})$, DIAD $(0.94 \mathrm{~mL}, 4.8 \mathrm{mmol})$ and phthalimide $(0.53 \mathrm{~g}, 3.6 \mathrm{mmol})$ at room temperature. The mixture was stirred for 3 hours and concentrated. The residue was purified by column chromatography over silica gel (DCM : $\left.\mathrm{MeOH}: \mathrm{NH}_{3} \mathrm{H}_{2} \mathrm{O}=200: 10: 0.1\right)$ to yield a brown oil, which was used directly for the next step.

To a stirred solution of above oil in EtOH $(50 \mathrm{~mL})$ was added hydrazine hydrate $(1 \mathrm{~mL})$ at room temperature. The mixture was refluxed for 2 hours and filtered. The filtrate was concentrated. The residue was purified by column chromatography over silica gel (DCM : $\left.\mathrm{MeOH}: \mathrm{NH}_{3} \mathrm{H}_{2} \mathrm{O}=100: 10: 0.2\right)$ to yield compound $4 \mathbf{a}(680 \mathrm{mg}, 68 \%)$ as a colorless oil, ${ }^{1} \mathrm{H}$ $\operatorname{NMR}\left(500 \mathrm{MHz}, \mathrm{CDCl}_{3}\right) \delta 8.44(\mathrm{~s}, 1 \mathrm{H}$, purin-H), $7.88(\mathrm{~s}, 1 \mathrm{H}$, purin- $\mathrm{H}), 7.35-7.30(\mathrm{~m}, 1 \mathrm{H}, \mathrm{Ar}-\mathrm{H})$, $7.19(\mathrm{~d}, \mathrm{~J}=7.5 \mathrm{~Hz}, 1 \mathrm{H}, \mathrm{Ar}-\mathrm{H}), 7.12(\mathrm{~d}, \mathrm{~J}=8.2 \mathrm{~Hz}, 1 \mathrm{H}, \mathrm{Ar}-\mathrm{H}), 7.0(\mathrm{t}, \mathrm{J}=5.1 \mathrm{~Hz}, 1 \mathrm{H}, \mathrm{Ar}-\mathrm{H}), 6.34$ (brs, $1 \mathrm{H}, \mathrm{NH}), 6.04(\mathrm{~d}, \mathrm{~J}=2.6 \mathrm{~Hz}, 1 \mathrm{H}$, tetrahydrofuro- $\mathrm{H}), 5.48-5.46(\mathrm{~m}, 1 \mathrm{H}$, tetrahydrofuro- $\mathrm{H})$, 5.16-5.14 $(\mathrm{m}, \quad 1 \mathrm{H}$, tetrahydrofuro- $\mathrm{H}), 4.91$ (brs, $2 \mathrm{H}$, benzyl- $\left.\mathrm{CH}_{2}\right), 4.36$ (brs, $1 \mathrm{H}$, tetrahydrofuro- $\mathrm{H}), 3.16-3.10\left(\mathrm{~m}, 2 \mathrm{H}, \mathrm{CH}_{2}\right), 1.64\left(\mathrm{~s}, 3 \mathrm{H}, \mathrm{CH}_{3}\right), 1.40\left(\mathrm{~s}, 3 \mathrm{H}, \mathrm{CH}_{3}\right) ;{ }^{13} \mathrm{C} \mathrm{NMR}(400$ $\left.\mathrm{MHz}, \mathrm{CDCl}_{3}\right) \delta 162.5(\mathrm{~d}, \mathrm{~J}=242.5 \mathrm{~Hz}$, Ar-C), 154.7 (purin-C), 153.2 (purin-C), $141.1(\mathrm{~d}, \mathrm{~J}=7.1$ $\mathrm{Hz}, \mathrm{Ar}-\mathrm{C}$ ), 140.0 (purin-C), 139.41 (purin-C), 130.1 (d, J = 8.2 Hz), 123.1 (d, J = 2.7 Hz, Ar-C), 120.5 (purin-C), 114.7 (acetonide-C), 114.5 (d, J = 21.6 Hz, Ar-C), 114.4 (d, J = 21.5 Hz, Ar-C), 91.2 (tetrahydrofuro-C), 86.1 (tetrahydrofuro-C), 83.5 (tetrahydrofuro-C), 81.6 (tetrahydrofuro-C), $43.3\left(\mathrm{CH}_{2}-\mathrm{C}\right), 41.8$ (benzyl-CH$\left.-\mathrm{C}\right), 27.2\left(\mathrm{CH}_{3}-\mathrm{C}\right), 25.3\left(\mathrm{CH}_{3}-\mathrm{C}\right)$; LRMS (ESI): $\mathrm{m} / \mathrm{z}=415[\mathrm{M}+$ $\mathrm{H}]^{+}$.

4.2.4.

9-((3aR,4R,6R,6aR)-6-(aminomethyl)-2,2-dimethyltetrahydrofuro[3,4-d][1,3]dioxol-4-yl)-N-(3-c hlorobenzyl)-9H-purin-6-amine $\mathbf{4 b}$

Following above synthetic procedure of compound $\mathbf{4 a}$, replacing $\mathbf{3 a}$ with $\mathbf{3 b}$ afforded compound $4 \mathbf{b}(75 \%)$ as a colorless oil, $[\alpha]^{20}{ }_{\mathrm{D}}=-23.43(\mathrm{c} 1.05, \mathrm{MeOH}) ;{ }^{1} \mathrm{H}$ NMR $(500 \mathrm{MHz}$, $\left.\mathrm{CDCl}_{3}\right) \delta 8.44(\mathrm{~s}, 1 \mathrm{H}$, purin-H), $7.88(\mathrm{~s}, 1 \mathrm{H}$, purin- $\mathrm{H}), 7.40(\mathrm{~s}, 1 \mathrm{H}, \mathrm{Ar}-\mathrm{H}), 7.35-7.30(\mathrm{~m}, 3 \mathrm{H}$, Ar-H), $6.34(\mathrm{brs}, 1 \mathrm{H}, \mathrm{NH}), 6.06(\mathrm{~d}, \mathrm{~J}=2.6 \mathrm{~Hz}, 1 \mathrm{H}$, tetrahydrofuro-H), 5.48-5.46 (m, $1 \mathrm{H}$, tetrahydrofuro- $\mathrm{H}), 5.16-5.14(\mathrm{~m}, 1 \mathrm{H}$, tetrahydrofuro- $\mathrm{H}), 4.91$ (brs, $2 \mathrm{H}$, benzyl- $\left.\mathrm{CH}_{2}\right), 4.30$ (brs, 
$1 \mathrm{H}$, tetrahydrofuro-H), 3.10-2.99 (m, 2H, $\left.\mathrm{CH}_{2}\right), 1.64\left(\mathrm{~s}, 3 \mathrm{H}, \mathrm{CH}_{3}\right), 1.43\left(\mathrm{~s}, 3 \mathrm{H}, \mathrm{CH}_{3}\right)$; LRMS (ESI): $\mathrm{m} / \mathrm{z}=431[\mathrm{M}+\mathrm{H}]^{+}$.

4.2.5. N-(3-fluorobenzyl)-9-((3aR,4R,6R,6aR)-6-((isopropylamino)methyl)-2,2-dimethyl tetrahydrofuro[3,4-d][1,3]dioxol-4-yl)-9H-purin-6-amine 5a

To a stirred solution of compound $\mathbf{4 a}(1 \mathrm{~g}, 2.4 \mathrm{mmol})$ in $\mathrm{MeOH}(30 \mathrm{~mL})$ was added acetone $(3 \mathrm{~mL})$ and $\mathrm{NaCNBH}_{3}(0.6 \mathrm{~g}, 9.6 \mathrm{mmol})$ at room temperature. The mixture was stirred for 15 minutes, adjusted to $\mathrm{pH} 7$ by acetic acid. The mixture was stirred 4 hours at the same temperature, quenched by $1 \mathrm{M} \mathrm{NaOH}$ solution $(10 \mathrm{~mL})$ at $0{ }^{\circ} \mathrm{C}$, diluted by $\mathrm{H}_{2} \mathrm{O}(50 \mathrm{~mL})$, and extracted by $\operatorname{DCM}(30 \mathrm{~mL} \times 3)$. The organic layer was washed by brine, dried over anhydrous $\mathrm{MgSO}_{4}$, filtered and concentrated. The residue was purified by column chromatography over silica gel (DCM : $\left.\mathrm{MeOH}: \mathrm{NH}_{3} \mathrm{H}_{2} \mathrm{O}=100: 10: 1\right)$ to yield compound $\mathbf{5 a}(850 \mathrm{mg}, 77 \%)$ as an oil, ${ }^{1} \mathrm{H}$ NMR $(500$ $\left.\mathrm{MHz}, \mathrm{CDCl}_{3}\right) \delta 8.43(\mathrm{~s}, 1 \mathrm{H}$, purin- $\mathrm{H}), 7.89(\mathrm{~s}, 1 \mathrm{H}$, purin- $\mathrm{H}), 7.35-7.30(\mathrm{~m}, 1 \mathrm{H}, \mathrm{Ar}-\mathrm{H}), 7.18(\mathrm{~d}, \mathrm{~J}$ $=7.5 \mathrm{~Hz}, 1 \mathrm{H}, \mathrm{Ar}-\mathrm{H}), 7.12(\mathrm{~d}, \mathrm{~J}=9.5 \mathrm{~Hz}, \mathrm{Ar}-\mathrm{H}), 7.01(\mathrm{t}, \mathrm{J}=7.0 \mathrm{~Hz}, 1 \mathrm{H}, \mathrm{Ar}-\mathrm{H}), 6.48(\mathrm{brs}, 1 \mathrm{H}$, $\mathrm{NH}), 6.05(\mathrm{~s}, 1 \mathrm{H}$, tetrahydrofuro-H), 5.51 (brs, $1 \mathrm{H}$, tetrahydrofuro- $\mathrm{H}), 5.10-5.08(\mathrm{~m}, 1 \mathrm{H}$, tetrahydrofuro- $\mathrm{H}$ ), 4.90 (brs, $2 \mathrm{H}$, benzyl- $\left.\mathrm{CH}_{2}\right), 4.40$ (brs, $1 \mathrm{H}$, tetrahydrofuro- $\left.\mathrm{H}\right), 2.98-2.90(\mathrm{~m}$, $\left.2 \mathrm{H}, \mathrm{CH}_{2}\right), 2.80-2.78(\mathrm{~m}, 1 \mathrm{H}$, isopropyl- $\mathrm{CH}), 1.65\left(\mathrm{~s}, 3 \mathrm{H}, \mathrm{CH}_{3}\right), 1.43\left(\mathrm{~s}, 1 \mathrm{H}, \mathrm{CH}_{3}\right), 1.08(\mathrm{~d}, \mathrm{~J}=6.2$ $\mathrm{Hz}, 3 \mathrm{H}$, isopropyl- $\left.\mathrm{CH}_{3}\right), 1.05\left(\mathrm{~d}, \mathrm{~J}=6.2 \mathrm{~Hz}, 3 \mathrm{H}\right.$, isopropyl- $\left.\mathrm{CH}_{3}\right) ;{ }^{13} \mathrm{C} \mathrm{NMR}\left(400 \mathrm{MHz}, \mathrm{CDCl}_{3}\right) \delta$ 162.5 (d, J = 242.5 Hz, Ar-C), 154.7 (purin-C), 153.2 (purin-C), 148.6 (purin-C), 141.1 (d, J = 6.8 $\mathrm{Hz}, \mathrm{Ar}-\mathrm{C}$ ), 139.4 (purin-C), 130.1 (d, J = 8.2 Hz, Ar-C), 123.0 (d, J = 2.7 Hz, Ar-C), 120.5 (purin-C), 114.6 (acetonide-C), 114.5 (d, J = 21.6 Hz, Ar-C), 114.4 (d, J = 21.5 Hz, Ar-C), 90.9 (tetrahydrofuro-C), 85.7 (tetrahydrofuro-C), 83.5 (tetrahydrofuro-C), 82.2 (tetrahydrofuro-C), 48.9 (isopropyl-CH-C), $48.8 \quad\left(\mathrm{CH}_{2}-\mathrm{C}\right), 43.9$ (benzyl-C), 27.3 (acetonide- $\mathrm{CH}_{3}-\mathrm{C}$ ), 25.4 (acetonide- $\mathrm{CH}_{3}-\mathrm{C}$ ), 22.7 (isopropyl- $\mathrm{CH}_{3}-\mathrm{C}$ ), 22.6 (isopropyl- $\mathrm{CH}_{3}-\mathrm{C}$ ); LRMS (ESI): $\mathrm{m} / \mathrm{z}=457$ [M $+\mathrm{H}]^{+}$.

4.2.6. N-(3-chlorobenzyl)-9-((3aR,4R,6R,6aR)-6-((isopropylamino)methyl)-2,2-dimethyl tetrahydrofuro[3,4-d][1,3]dioxol-4-yl)-9H-purin-6-amine 5b

Following above synthetic procedure of compound 5a, replacing $\mathbf{4 a}$ with $\mathbf{4 b}$ afforded compound 5b (75\%) as an oil, LRMS (ESI): $\mathrm{m} / \mathrm{z}=473[\mathrm{M}+\mathrm{H}]^{+}$.

4.2.7.

methyl

3-((((3aR,4R,6R,6aR)-6-(6-((3-fluorobenzyl)amino)-9H-purin-9-yl)-2,2-dimethyltetrahydrofuro[3 ,4-d][1,3]dioxol-4-yl)methyl)(isopropyl)amino)propanoate 6a

A solution of $\mathbf{5 a}(1 \mathrm{~g}, 2.2 \mathrm{mmol})$ and methyl acrylate $(0.4 \mathrm{~mL})$ in $\mathrm{MeOH}(10 \mathrm{~mL})$ was sealed in a microwave tube, heated to $90{ }^{\circ} \mathrm{C}$ under microwave radiation for 3 hours. The mixture was concentrated and purified by column chromatography over silica gel (DCM : $\mathrm{MeOH}=40: 1)$ to yield compound 6a $(0.9 \mathrm{~g}, 76 \%)$ as an oil, ${ }^{1} \mathrm{H}$ NMR $\left(500 \mathrm{MHz}, \mathrm{CDCl}_{3}\right) \delta 8.43(\mathrm{~s}, 1 \mathrm{H}$, purin- $\mathrm{H})$, $7.90(\mathrm{~s}, 1 \mathrm{H}$, purin-H), 7.35-7.30 (m, 1H, Ar-H), $7.18(\mathrm{~d}, \mathrm{~J}=7.5 \mathrm{~Hz}, 1 \mathrm{H}, \operatorname{Ar}-\mathrm{H}), 7.12(\mathrm{~d}, \mathrm{~J}=9.5$ $\mathrm{Hz}, \mathrm{Ar}-\mathrm{H}), 7.01(\mathrm{t}, \mathrm{J}=7.0 \mathrm{~Hz}, 1 \mathrm{H}, \mathrm{Ar}-\mathrm{H}), 6.32$ (brs, $1 \mathrm{H}, \mathrm{NH}), 6.07$ (s, 1H, tetrahydrofuro-H), 5.57 (brs, $1 \mathrm{H}$, tetrahydrofuro- $\mathrm{H}$ ), 5.08 (brs, $1 \mathrm{H}$, tetrahydrofuro- $\mathrm{H}$ ), 4.90 (brs, $2 \mathrm{H}$, benzyl- $\mathrm{CH}_{2}$ ), 4.34 (brs, $1 \mathrm{H}$, tetrahydrofuro- $\mathrm{H}), 3.69\left(\mathrm{~s}, 3 \mathrm{H}, \mathrm{MeO}-\mathrm{CH}_{3}\right), 2.94-2.48\left(\mathrm{~m}, 7 \mathrm{H}, \mathrm{CH}_{2} \mathrm{CH}_{2}, \mathrm{CH}_{2}\right.$, and isopropyl- $\mathrm{CH}), 1.65\left(\mathrm{~s}, 3 \mathrm{H}\right.$, acetonide- $\left.\mathrm{CH}_{3}\right), 1.43\left(\mathrm{~s}, 3 \mathrm{H}\right.$, acetonide- $\left.\mathrm{CH}_{3}\right), 1.05(\mathrm{~s}, 3 \mathrm{H}$, isopropyl- $\left.\mathrm{CH}_{3}\right), 0.92\left(\mathrm{~s}, 3 \mathrm{H}\right.$, isopropyl- $\left.\mathrm{CH}_{3}\right) ;{ }^{13} \mathrm{C} \mathrm{NMR}\left(400 \mathrm{MHz}, \mathrm{CDCl}_{3}\right) \delta 162.5(\mathrm{~d}, \mathrm{~J}=242.5$ $\mathrm{Hz}, \mathrm{Ar}-\mathrm{C}$ ), 154.7 (purin-C), 153.2 (purin-C), 148.6 (purin-C), 141.1 (d, J = 6.8 Hz, Ar-C), 139.8 (purin-C), 130.1 (d, J = 8.2 Hz, Ar-C), 123.0 (d, J = 2.7 Hz, Ar-C), 120.5 (purin-C), 114.5 (d, J = $21.6 \mathrm{~Hz}, \mathrm{Ar}-\mathrm{C}$ ), 114.4 (d, J = 21.5 Hz, Ar-C), 91.2 (tetrahydrofuro-C), 83.6 (tetrahydrofuro-C), 83.2 (tetrahydrofuro-C), 77.2 (tetrahydrofuro-C), 51.9 (MeO-C), $51.7\left(\mathrm{CH}_{2}-\mathrm{C}\right), 46.3\left(\mathrm{CH}_{2}-\mathrm{C}\right)$, 
43.8 (benzyl- $\mathrm{CH}_{2}$ ), 27.1 (acetonide- $\mathrm{CH}_{3}-\mathrm{C}$ ), 25.4 (acetonide- $\mathrm{CH}_{3}-\mathrm{C}$ ), 18.8 (isopropyl- $\mathrm{CH}_{3}-\mathrm{C}$ ), 16.7 (isopropyl- $\mathrm{CH}_{3}-\mathrm{C}$ ); LRMS (ESI): $\mathrm{m} / \mathrm{z}=543[\mathrm{M}+\mathrm{H}]^{+}$.

4.2.8.

methyl

3-((((3aR,4R,6R,6aR)-6-(6-((3-fluorobenzyl)amino)-9H-purin-9-yl)-2,2-dimethyltetrahydrofuro[3 ,4-d][1,3]dioxol-4-yl)methyl)(isopropyl)amino)propanoate $\mathbf{6 b}$

Following above synthetic procedure of compound $\mathbf{6 a}$, replacing $\mathbf{5 a}$ with $\mathbf{5 b}$ afforded compound $6 \mathbf{b}(80 \%)$ as an oil, LRMS (ESI): $\mathrm{m} / \mathrm{z}=559[\mathrm{M}+\mathrm{H}]^{+}$.

4.2.9.

3-((((3aR,4R,6R,6aR)-6-(6-((3-fluorobenzyl)amino)-9H-purin-9-yl)-2,2-dimethyl tetrahydrofuro[3,4-d][1,3]dioxol-4-yl)methyl)(isopropyl)amino)propan-1-ol 7a

To a stirred solution of $\mathbf{6 a}(900 \mathrm{mg}, 1.66 \mathrm{mmol})$ in anhydrous THF $(10 \mathrm{~mL})$ was added $\mathrm{LiAlH}_{4}\left(3.3 \mathrm{~mL}, 1 \mathrm{~N}\right.$ solution in THF) at $0{ }^{\circ} \mathrm{C}$. The mixture was stirred for 1 hour at $0{ }^{\circ} \mathrm{C}$, diluted by ether $(20 \mathrm{~mL})$, and slowly quenched by $\mathrm{H}_{2} \mathrm{O}(20 \mu \mathrm{L}), \mathrm{NaOH}$ solution $(20 \mu \mathrm{L}, 15 \%$ solution $)$, and $\mathrm{H}_{2} \mathrm{O}(60 \mu \mathrm{L})$ succesively. The mixture was stirred for 15 minutes. To the mixture was added anhydrous $\mathrm{MgSO}_{4}$, filtered and concentrated. The residue was purified by column chromatography over silica gel (DCM : $\mathrm{MeOH}=40: 1)$ to yield compound $7 \mathbf{a}(680 \mathrm{mg}, 80 \%)$ as an oil, ${ }^{1} \mathrm{H}$ NMR $\left(500 \mathrm{MHz}, \mathrm{CDCl}_{3}\right) \delta 8.43(\mathrm{~s}, 1 \mathrm{H}$, purin-H), $7.92(\mathrm{~s}, 1 \mathrm{H}$, purin-H), 7.35-7.30 (m, $1 \mathrm{H}, \operatorname{Ar}-\mathrm{H}), 7.18(\mathrm{~d}, \mathrm{~J}=7.5 \mathrm{~Hz}, 1 \mathrm{H}, \operatorname{Ar}-\mathrm{H}), 7.12(\mathrm{~d}, \mathrm{~J}=9.5 \mathrm{~Hz}, \operatorname{Ar}-\mathrm{H}), 7.01(\mathrm{t}, \mathrm{J}=7.0 \mathrm{~Hz}, 1 \mathrm{H}$, Ar-H), 6.28 (brs, $1 \mathrm{H}, \mathrm{NH}), 6.12$ (s, 1H, tetrahydrofuro- $\mathrm{H}), 5.54$ (brs, $1 \mathrm{H}$, tetrahydrofuro- $\mathrm{H}), 5.11$ (brs, $1 \mathrm{H}$, tetrahydrofuro- $\mathrm{H}$ ), 4.92 (brs, $2 \mathrm{H}$, benzyl- $\left.\mathrm{CH}_{2}\right), 3.79-3.73\left(\mathrm{~m}, 3 \mathrm{H}, \mathrm{CH}_{2}\right.$ and $\left.\mathrm{CH}\right)$, 3.10-2.78 (m, $\left.4 \mathrm{H}, \mathrm{CH}_{2} \mathrm{CH}_{2}\right), 1.82-1.78\left(\mathrm{~m}, 2 \mathrm{H}, \mathrm{CH}_{2}\right), 1.66\left(\mathrm{~s}, 3 \mathrm{H}\right.$, acetonide- $\left.\mathrm{CH}_{3}\right), 1.43(\mathrm{~s}, 3 \mathrm{H}$, acetonide- $\left.\mathrm{CH}_{3}\right), 1.12\left(\mathrm{~s}, 3 \mathrm{H}\right.$, isopropyl- $\left.\mathrm{CH}_{3}\right), 0.87\left(\mathrm{~s}, 3 \mathrm{H}\right.$, isopropyl- $\left.\mathrm{CH}_{3}\right) ;{ }^{13} \mathrm{C} \mathrm{NMR}(400 \mathrm{MHz}$, $\mathrm{CDCl}_{3}$ ) $\delta 162.5(\mathrm{~d}, \mathrm{~J}=242.5 \mathrm{~Hz}$, Ar-C), 154.7 (purin-C), 153.2 (purin-C), 148.6 (purin-C), 141.1 $(\mathrm{d}, \mathrm{J}=6.8 \mathrm{~Hz}, \mathrm{Ar}-\mathrm{C}$ ), 140.1 (purin-C), 130.1 (d, J = 8.2 Hz, Ar-C), 123.0 (d, J = 2.7 Hz, Ar-C), 120.5 (purin-C), 114.9 (acetonide-C), 114.5 (d, J = 21.6 Hz, Ar-C), 114.4 (d, J = 21.5 Hz, Ar-C), 90.7 (tetrahydrofuro-C), 83.8 (tetrahydrofuro-C), 83.4 (tetrahydrofuro-C), 77.2 (tetrahydrofuro-C), 65.6 (tetrahydrofuro-C), $62.7 \quad\left(\mathrm{CH}_{2} \mathrm{OH}-\mathrm{C}\right), 51.4$ (isopropyl-CH-C), $49.3 \quad\left(\mathrm{CH}_{2}-\mathrm{C}\right), 43.8$ (benzyl-CH2-C), 27.1 (acetonide- $\mathrm{CH}_{3}-\mathrm{C}$ ), 25.4 (acetonide- $\mathrm{CH}_{3}-\mathrm{C}$ ), 17.6 (isopropyl- $\mathrm{CH}_{3}-\mathrm{C}$ ), 15.8 (isopropyl- $\mathrm{CH}_{3}-\mathrm{C}$ ); LRMS (ESI): $\mathrm{m} / \mathrm{z}=515[\mathrm{M}+\mathrm{H}]^{+}$.

4.2.10. 3- 3-(((3aR,4R,6R,6aR)-6-(6-((3-chlorobenzyl)amino)-9H-purin-9-yl)-2,2-dimethyl tetrahydrofuro[3,4-d][1,3]dioxol-4-yl)methyl)(isopropyl)amino)propan-1-ol 7b

Following above synthetic procedure of compound $7 \mathbf{a}$, replacing $6 \mathbf{a}$ with $\mathbf{6 b}$ afforded compound $7 \mathbf{b}(85 \%)$ as an oil, LRMS (ESI): $\mathrm{m} / \mathrm{z}=531[\mathrm{M}+\mathrm{H}]^{+}$.

4.2.11.

N1-(((3aR,4R,6R,6aR)-6-(6-((3-fluorobenzyl)amino)-9H-purin-9-yl)-2,2-dimethyltetrahydrofuro[ 3,4-d][1,3]dioxol-4-yl)methyl)-N1-isopropylpropane-1,3-diamine 8a

Following above synthetic procedure of compound $\mathbf{4 a}$, replacing $\mathbf{3 a}$ with $\mathbf{7 a}$ afforded crude $\mathbf{8 a}$ (80\% percent pure from LC-MS, yield 76\%) which was used directly for the next step without further purification, LRMS (ESI): $\mathrm{m} / \mathrm{z}=514[\mathrm{M}+\mathrm{H}]^{+}$.

4.2.12.

N1-(((3aR,4R,6R,6aR)-6-(6-((3-fluorobenzyl)amino)-9H-purin-9-yl)-2,2-dimethyltetrahydrofuro[ 3,4-d][1,3]dioxol-4-yl)methyl)-N1-isopropylpropane-1,3-diamine $\mathbf{8 b}$ 
Following above synthetic procedure of compound $\mathbf{4 a}$, replacing $\mathbf{3 a}$ with $\mathbf{7 b}$ afforded $\mathbf{8 b}$ (yield $78 \%$ ) as an oil which was used directly for the next step without further purification, $[\alpha]^{20}$ $=-2.15(\mathrm{c} 1.35, \mathrm{MeOH}) ;{ }^{1} \mathrm{H}$ NMR $\left(500 \mathrm{MHz}, \mathrm{CDCl}_{3}\right) \delta 8.41(\mathrm{~s}, 1 \mathrm{H}$, purin- $\mathrm{H}), 8.01(\mathrm{~s}, 1 \mathrm{H}$, purin-H), $7.37(\mathrm{~s}, 1 \mathrm{H}, \mathrm{Ar}-\mathrm{H}), 7.30-7.28(\mathrm{~m}, 3 \mathrm{H}, \mathrm{Ar}-\mathrm{H}), 6.66$ (brs, $1 \mathrm{H}, \mathrm{NH}), 6.12(\mathrm{~s}, 1 \mathrm{H}$, tetrahydrofuran-H), $5.59(\mathrm{~d}, \mathrm{~J}=5.8 \mathrm{~Hz}, 1 \mathrm{H}$, tetrahydrofuran- $\mathrm{H}), 5.05-5.03(\mathrm{~m}, 1 \mathrm{H}$, tetrahydrofuran- $\mathrm{H}), 4.87$ (brs, $2 \mathrm{H}$, benzyl- $\left.\mathrm{CH}_{2}\right), 4.32$ (brs, $1 \mathrm{H}$, tetrahydrofuran- $\left.\mathrm{H}\right), 2.97-2.95(\mathrm{~m}$, $1 \mathrm{H}$, isopropyl-CH), 2.81-2.79 (m, $\left.2 \mathrm{H}, \mathrm{CH}_{2}\right), 2.68-2.65\left(\mathrm{~m}, 1 \mathrm{H}, \mathrm{CH}_{2}-\mathrm{H}\right), 2.53-2.50(\mathrm{~m}, 1 \mathrm{H}$, $\left.\mathrm{CH}_{2}-\mathrm{H}\right), 1.82-1.78\left(\mathrm{~m}, 2 \mathrm{H}, \mathrm{CH}_{2}\right), 1.59-1.54\left(\mathrm{~m}, 2 \mathrm{H}, \mathrm{CH}_{2}\right), 1.42(\mathrm{~s}, 3 \mathrm{H}),, 0.98(\mathrm{~d}, \mathrm{~J}=6.5 \mathrm{~Hz}, 3 \mathrm{H}$, $\left.\mathrm{CH}_{3}\right), 0.78\left(\mathrm{~d}, \mathrm{~J}=6.5 \mathrm{~Hz}, 3 \mathrm{H}, \mathrm{CH}_{3}\right) ; \mathrm{LRMS}(\mathrm{ESI}): \mathrm{m} / \mathrm{z}=530[\mathrm{M}+\mathrm{H}]^{+}$.

\subsubsection{General procedure for the synthesis of SIN derivatives 1aa-ah, $\mathbf{1 b a - b h}$.}

To a stirred solution of $\mathbf{8 a - b}(0.15 \mathrm{mmol})$ in acetonitrile $(5 \mathrm{~mL})$ was added isocyanate $(0.2$ $\mathrm{mmol}$ ) at room temperature. The mixture was stirred for 1 hour and concentrated. The residue was purified by column chromatography over silica gel ( $\mathrm{DCM}: \mathrm{MeOH}=20: 1)$ to yield an oil.

To a stirred solution of above solid in DCM $(6 \mathrm{~mL})$ was added TFA $(1 \mathrm{~mL})$ and $\mathrm{H}_{2} \mathrm{O}(0.5 \mathrm{~mL})$ at $0{ }^{\circ} \mathrm{C}$. The mixture was stirred overnight and concentrated. The residue was purified by preparing TLC (DCM : $\left.\mathrm{MeOH}: \mathrm{NH}_{3} \mathrm{H}_{2} \mathrm{O}=70: 10: 1\right)$ to yield SIN derivatives 1aa-ah, 1ba-bh.

4.2.13.1. 1-(3-((((2R,3S,4R,5R)-5-(6-((3-fluorobenzyl)amino)-9H-purin-9-yl)-3,4-dihydroxy tetrahydrofuran-2-yl)methyl)(isopropyl)amino)propyl)-3-(4-fluorophenyl)urea 1aa

According to the general procedure, employing 8a and 1-fluoro-4- isocyanatobenzene afforded compound 1aa as a solid, 80\% yield, HPLC purity: $97.6 \%$, method A; mp: $180-182{ }^{\circ} \mathrm{C}$; $[\alpha]^{20}{ }_{\mathrm{D}}=12.91(\mathrm{c} 0.55, \mathrm{MeOH}) ;{ }^{1} \mathrm{H}$ NMR $(500 \mathrm{MHz}, \mathrm{MeOD}) \delta 8.28(\mathrm{~s}, 1 \mathrm{H}$, purin-H), $8.24(\mathrm{~s}, 1 \mathrm{H}$, purin-H), 7.35-7.29 (m, 3H, Ar-H), $7.22(\mathrm{~d}, \mathrm{~J}=7.5 \mathrm{~Hz}, 1 \mathrm{H}, \mathrm{Ar}-\mathrm{H}), 7.15$ (d, J = 9.8 Hz, 1H, Ar-H), 7.01-6.95 (m, 3H, Ar-H), 6.02 (d, J = 4.0 Hz, 1H, tetrahydrofuran-H), 4.88 (brs, 2H, benzyl- $\mathrm{CH}_{2}$ ), 4.77 (brs, $1 \mathrm{H}$, tetrahydrofuran $-\mathrm{H}), 4.37-4.35(\mathrm{~m}, 1 \mathrm{H}$, tetrahydrofuran- $\mathrm{H}), 4.25-4.22(\mathrm{~m}, 1 \mathrm{H}$, tetrahydrofuran- $\mathrm{H}), 3.28-3.21\left(\mathrm{~m}, 3 \mathrm{H}, \mathrm{CH}_{2}\right.$ and isoproyly- $\left.\mathrm{CH}\right), 3.08-2.76\left(\mathrm{~m}, 4 \mathrm{H}, \mathrm{CH}_{2}\right.$ and $\left.\mathrm{CH}_{2}\right)$, 1.76-1.73 (m, $\left.2 \mathrm{H}, \mathrm{CH}_{2}\right), 1.15$ (s, $3 \mathrm{H}$, isopropyl- $\left.\mathrm{CH}_{3}\right), 1.10$ (s, $3 \mathrm{H}$, isopropyl- $\left.\mathrm{CH}_{3}\right) ;{ }^{13} \mathrm{C} \mathrm{NMR}(400$ MHz, MeOD) $\delta 162.1(\mathrm{~d}, \mathrm{~J}=244.5 \mathrm{~Hz}, \mathrm{~F}-$ phenyl-C), 158.6 (d, J = 240.1 Hz, F-phenyl-C), 156.9 (urea-C), 154.5 (purin-C), 152.4 (purin-C), 141.9 (purin-C), 139.7 (purin-C), 135.5 (3-F-phenyl-C), 129.7 (d, J = 8.3 Hz, Ar-C), 129.3 (Ar-C), 122.7 (d, J = 2.8 Hz, Ar-C), 120.6 (d, J = 7.7 Hz, Ar-C), 119.6 (purin-C), 114.7 (d, J = 22.6 Hz, 4-F-phenyl-C), 113.6 (d, J = 20.6 Hz, 3-F-phenyl-C), 113.3 (d, $\mathbf{J}=20.3 \mathrm{~Hz}, 3-\mathrm{F}-$ phenyl-C), 89.2 (tetrahydrofuran-C), 82.7 (tetrahydrofuran-C), 73.2 (tetrahydrofuran-C), 72.1 (tetrahydrofuran-C), $52.1\left(\mathrm{CH}_{2}-\mathrm{C}\right), 43.0$ (benzyl- $\mathrm{CH}_{2}-\mathrm{C}$ ), $37.7\left(\mathrm{CH}_{2}-\mathrm{C}\right), 27.3\left(\mathrm{CH}_{2}-\mathrm{C}\right), 16.5$ (isopropyl- $\left.\mathrm{CH}_{3}\right), 16.0$ (isopropyl- $\left.\mathrm{CH}_{3}\right)$; LRMS (ESI): $\mathrm{m} / \mathrm{z}=633[\mathrm{M}+\mathrm{Na}]^{+}$; HRMS-ESI $(\mathrm{m} / \mathrm{z})$ : Calcd. For $\mathrm{C}_{30} \mathrm{H}_{37} \mathrm{~F}_{2} \mathrm{~N}_{8} \mathrm{O}_{4}(\mathrm{M}+\mathrm{H})^{+}$: 611.2900; Found: 611.2897.

\subsubsection{2.}

1-(3-((((2R,3S,4R,5R)-5-(6-((3-fluorobenzyl)amino)-9H-purin-9-yl)-3,4-dihydroxytetrahydrofura n-2-yl)methyl)(isopropyl)amino)propyl)-3-(p-tolyl)urea 1ab

According to the general procedure, employing 8a and 1-isocyanato-4-methylbenzene afforded compound 1ab as a solid, $76 \%$ yield, HPLC purity: $98.9 \%$, method A; mp: $190-192{ }^{\circ} \mathrm{C} ;[\alpha]^{20}{ }_{\mathrm{D}}=$ $17.36(\mathrm{c} 0.67, \mathrm{MeOH}) ;{ }^{1} \mathrm{H}$ NMR $(500 \mathrm{MHz}, \mathrm{MeOD}) \delta 8.28(\mathrm{~s}, 1 \mathrm{H}$, purin-H), $8.20(\mathrm{~s}, 1 \mathrm{H}$, purin-H), 7.36-7.30 (m, 1H, F-phenyl-H), 7.22-7.18 (m, 3H, Ar-H), $7.13(\mathrm{~d}, \mathrm{~J}=9.8 \mathrm{~Hz}, 1 \mathrm{H}$, F-phenyl-H), 7.04 (d, J = 7.6 Hz, 1H, tolyl-H), $6.96(\mathrm{t}, \mathrm{J}=7.5 \mathrm{~Hz}, 1 \mathrm{H}, \mathrm{F}-$ phenyl-H), 6.02 (d, J = $4.0 \mathrm{~Hz}, 1 \mathrm{H}$, tetrahydrofuran- $\mathrm{H}), 4.88$ (brs, $2 \mathrm{H}$, benzyl- $\left.\mathrm{CH}_{2}\right), 4.77$ (brs, $1 \mathrm{H}$, tetrahydrofuran- $\mathrm{H}$ ), 4.41-4.38 $(\mathrm{m}, 1 \mathrm{H}$, tetrahydrofuran- $\mathrm{H}), 4.30-4.25(\mathrm{~m}, 1 \mathrm{H}$, tetrahydrofuran- $\mathrm{H}), 3.44-3.41(\mathrm{~m}, 1 \mathrm{H}$, isopropyl- $\mathrm{CH}$ ), 3.26-3.20 (m, $4 \mathrm{H}, \mathrm{CH}_{2}$ and $\left.\mathrm{CH}_{2}\right), 2.92-2.90\left(\mathrm{~m}, 2 \mathrm{H}, \mathrm{CH}_{2}\right), 2.72\left(\mathrm{~s}, 3 \mathrm{H}\right.$, tolyl- $\left.\mathrm{CH}_{3}\right)$, 1.82-1.78 (m, $\left.2 \mathrm{H}, \mathrm{CH}_{2}\right), 1.22\left(\mathrm{~s}, 3 \mathrm{H}\right.$, isopropyl- $\left.\mathrm{CH}_{3}\right), 1.17\left(\mathrm{~s}, 3 \mathrm{H}\right.$, isopropyl- $\left.\mathrm{CH}_{3}\right) ;{ }^{13} \mathrm{C}$ NMR $(400$ 
$\mathrm{MHz}, \mathrm{MeOD}) \delta 162.1$ (d, J = 244.5 Hz, F-phenyl-C), 157.3 (urea-C), 154.6 (purin-C), 152.4 (purin-C), 141.9 (purin-C), 139.8 (purin-C), 136.5 (Me-phenyl-C), 131.7 (Me-phenyl-C), 129.8 (d, $\mathrm{J}=8.3 \mathrm{~Hz}$, F-phenyl-C), 128.7 (Me-phenyl-C), 122.7 (d, J = 2.8 Hz, F-phenyl-C), 119.8 (purin-C), 119.1 (Me-phenyl-C), $113.6(\mathrm{~d}, \mathrm{~J}=22.1 \mathrm{~Hz}, \mathrm{~F}-$ phenyl-C), $113.3(\mathrm{~d}, \mathrm{~J}=21.9 \mathrm{~Hz}$, F-phenyl-C), 89.7 (tetrahydrofuran-C), 81.5 (tetrahydrofuran-C), 73.1 (tetrahydrofuran-C), 72.0 (tetrahydrofuran-C), $52.0\left(\mathrm{CH}_{2}-\mathrm{C}\right), 48.3\left(\mathrm{CH}_{2}-\mathrm{C}\right), 43.0$ (benzyl- $\left.\mathrm{CH}_{2}-\mathrm{C}\right), 37.2$ (isopropyl-CH-C), $26.8\left(\mathrm{CH}_{2}-\mathrm{C}\right.$ ), 16.1 (isopropyl- $\mathrm{CH}_{3}-\mathrm{C}$ ), 15.7 (isopropyl- $\mathrm{CH}_{3}-\mathrm{C}$ ); LRMS (ESI): $\mathrm{m} / \mathrm{z}=607$ [M + $\mathrm{H}]^{+}$; HRMS-ESI (m/z): Calcd. For $\mathrm{C}_{31} \mathrm{H}_{40} \mathrm{~N}_{8} \mathrm{O}_{4} \mathrm{~F}(\mathrm{M}+\mathrm{H})^{+}$: 607.3151; Found: 607.3129.

\subsubsection{3}

1-(3-((((2R,3S,4R,5R)-5-(6-((3-fluorobenzyl)amino)-9H-purin-9-yl)-3,4-dihydroxytetrahydrofura n-2-yl)methyl)(isopropyl)amino)propyl)-3-(4-methoxyphenyl)urea 1ac

According to the general procedure, employing 8a and 1-isocyanato-4-methoxylbenzene afforded compound 1ac as a solid, 79\% yield, HPLC purity: $98.4 \%$, method A; mp: $143-144{ }^{\circ} \mathrm{C}$; $[\alpha]^{20}{ }_{\mathrm{D}}=14.34(\mathrm{c} 0.45, \mathrm{MeOH}) ; \quad{ }^{1} \mathrm{H}$ NMR $\left(500 \mathrm{MHz}, \mathrm{CDCl}_{3}\right) \delta 8.30(\mathrm{~s}, 1 \mathrm{H}$, purin-H), $7.92(\mathrm{~s}$, $1 \mathrm{H}$, purin-H), $7.66(\mathrm{~s}, 1 \mathrm{H}, \mathrm{NH}), 7.29-7.24(\mathrm{~m}, 1 \mathrm{H}, \operatorname{Ar}-\mathrm{H}), 7.21(\mathrm{~d}, \mathrm{~J}=8.5 \mathrm{~Hz}, 2 \mathrm{H}$, MeO-phenyl-H), 7.14 (d, J = 7.5 Hz, 1H, F-phenyl-H), 7.09 (d, J = 9.5 Hz, 1H, F-phenyl-H), 6.95 $(\mathrm{t}, \mathrm{J}=7.5 \mathrm{~Hz}, 1 \mathrm{H}, \mathrm{F}-$ phenyl-H), $6.75(\mathrm{~d}, \mathrm{~J}=8.5 \mathrm{~Hz}, 2 \mathrm{H}, \mathrm{MeO}-$ phenyl-H), 6.66 (brs, $1 \mathrm{H}, \mathrm{NH})$, 6.31 (brs, $1 \mathrm{H}, \mathrm{NH}), 5.95\left(\mathrm{~d}, \mathrm{~J}=3.3 \mathrm{~Hz}, 1 \mathrm{H}\right.$, tetrahydrofuran- $\mathrm{H}$ ), 4.84 (brs, $2 \mathrm{H}$, benzyl- $\mathrm{CH}_{2}$ ), 4.65 (brs, $1 \mathrm{H}$, tetrahydrofuran- $\mathrm{H}), 4.51$ (brs, $1 \mathrm{H}$, tetrahydrofuran- $\mathrm{H}), 4.32$ (brs, $1 \mathrm{H}$, tetrahydrofuran- $\mathrm{H}$ ), $3.72\left(\mathrm{~s}, 3 \mathrm{H}, \mathrm{MeO}-\mathrm{CH}_{3}\right), 3.24-3.17\left(\mathrm{~m}, 3 \mathrm{H}, \mathrm{CH}_{2}\right.$ and isopropyl- $\left.\mathrm{CH}\right), 2.97-2.83\left(\mathrm{~m}, 2 \mathrm{H}, \mathrm{CH}_{2}\right), 2.67$ (brs, $\left.2 \mathrm{H}, \mathrm{CH}_{2}\right), 1.69-1.65\left(\mathrm{~m}, 2 \mathrm{H}, \mathrm{CH}_{2}\right), 1.07\left(\mathrm{~d}, \mathrm{~J}=5.6 \mathrm{~Hz}, 3 \mathrm{H}\right.$, isopropyl- $\left.\mathrm{CH}_{3}\right), 0.98(\mathrm{~d}, \mathrm{~J}=5.6$ $\mathrm{Hz}, 3 \mathrm{H}$, isopropyl- $\left.\mathrm{CH}_{3}\right) ;{ }^{13} \mathrm{C}$ NMR $\left(400 \mathrm{MHz}, \mathrm{CDCl}_{3}\right) \delta 162.1$ (d, J = 244.5 Hz, F-phenyl-C), 157.4 (MeO-phenyl-C), 155.8 (urea-C), 154.6 (purin-C), 152.9 (purin-C), 141.2 (purin-C), 139.0 (purin-C), 131.8 (MeO-phenyl-C), 130.1 (d, J = 8.3 Hz, F-phenyl-C), 123.1 (Ar-C), 122.3 (Ar-C), 120.1 (purin-C), 114.4 (d, J = 22.3 Hz, F-phenyl-C), 114.3 (MeO-phenyl-C), 114.1 (d, J = 22.0 Hz, F-phenyl-C), 89.8 (tetrahydrofuran-C), 82.0 (tetrahydrofuran-C), 74.1 (tetrahydrofuran-C), 72.5 (tetrahydrofuran-C), $55.4\left(\mathrm{MeO}-\mathrm{CH}_{3}\right), 51.9\left(\mathrm{CH}_{2}-\mathrm{C}\right), 48.4\left(\mathrm{CH}_{2}-\mathrm{C}\right), 43.8$ (benzyl- $\left.\mathrm{CH}_{2}-\mathrm{C}\right)$, 38.1 (isopropyl-CH-C), $29.7 \quad\left(\mathrm{CH}_{2}-\mathrm{C}\right), \quad 26.8 \quad\left(\mathrm{CH}_{2}-\mathrm{C}\right), \quad 17.9 \quad$ (isopropyl- $\left.\mathrm{CH}_{3}-\mathrm{C}\right), \quad 16.2$ (isopropyl- $\mathrm{CH}_{3}-\mathrm{C}$ ); LRMS (ESI): $\mathrm{m} / \mathrm{z}=645[\mathrm{M}+\mathrm{Na}]^{+}$; HRMS-ESI $(\mathrm{m} / \mathrm{z}$ ): Calcd. For $\mathrm{C}_{31} \mathrm{H}_{40} \mathrm{~N}_{8} \mathrm{O}_{5} \mathrm{~F}(\mathrm{M}+\mathrm{H})^{+}: 623.3100$; Found: 623.3078 .

\subsubsection{4.}

1-(4-(tert-butyl)phenyl)-3-(3-((((2R,3S,4R,5R)-5-(6-((3-fluorobenzyl)amino)-9H-purin-9-yl)-3,4dihydroxytetrahydrofuran-2-yl)methyl)(isopropyl)amino)propyl)urea 1ad

According to the general procedure, employing 8a and 1-(tert-butyl)-4-isocyanatobenzene afforded compound 1ad as a solid, $72 \%$ yield, HPLC purity: $96.5 \%$, method $\mathrm{B}$; mp: $110-112{ }^{\circ} \mathrm{C}$; $[\alpha]^{20}{ }_{\mathrm{D}}=10.12(\mathrm{c} 0.57, \mathrm{MeOH}) ; \quad{ }^{1} \mathrm{H}$ NMR $\left(500 \mathrm{MHz}, \mathrm{CDCl}_{3}\right) \delta 8.32(\mathrm{~s}, 1 \mathrm{H}$, purin-H), $7.94(\mathrm{~s}$, 1H, purin-H), $7.71(\mathrm{~s}, 1 \mathrm{H}, \mathrm{NH}), 7.29-7.24(\mathrm{~m}, 5 \mathrm{H}, \mathrm{Ar}-\mathrm{H}), 7.14$ (d, J = 7.5 Hz, 1H, F-phenyl-H), 7.09 (d, J = 9.5 Hz, 1H, F-phenyl-H), 6.96 (t, J = 7.5 Hz, 1H, F-phenyl-H), 6.66 (brs, 1H, NH), 6.36 (brs, $1 \mathrm{H}, \mathrm{NH}), 5.95$ (d, J = 3.3 Hz, $1 \mathrm{H}$, tetrahydrofuran-H), 4.85 (brs, $2 \mathrm{H}$, benzyl- $\mathrm{CH}_{2}$ ), 4.66 (brs, $1 \mathrm{H}$, tetrahydrofuran- $\mathrm{H}$ ), 4.54 (brs, $1 \mathrm{H}$, tetrahydrofuran- $\mathrm{H}$ ), 4.35 (brs, $1 \mathrm{H}$, tetrahydrofuran- $\mathrm{H}$ ), 3.27 (brs, $2 \mathrm{H}, \mathrm{CH}_{2}$ ), 3.14-3.10 (m, $1 \mathrm{H}$, isopropyl- $\mathrm{CH}$ ), 2.97-2.83 (m, $\left.2 \mathrm{H}, \mathrm{CH}_{2}\right), 2.67$ (brs, $2 \mathrm{H}$, $\left.\mathrm{CH}_{2}\right), 1.71-1.68\left(\mathrm{~m}, 2 \mathrm{H}, \mathrm{CH}_{2}\right), 1.27(\mathrm{~s}, 9 \mathrm{H}, \mathrm{tBu}), 1.07\left(\mathrm{~d}, \mathrm{~J}=5.6 \mathrm{~Hz}, 3 \mathrm{H}\right.$, isopropyl- $\left.\mathrm{CH}_{3}\right), 0.98(\mathrm{~d}$, $\mathrm{J}=5.6 \mathrm{~Hz}, 3 \mathrm{H}$, isopropyl- $\left.\mathrm{CH}_{3}\right) ;{ }^{13} \mathrm{C} \mathrm{NMR}\left(400 \mathrm{MHz}, \mathrm{CDCl}_{3}\right) \delta 162.1(\mathrm{~d}, \mathrm{~J}=244.5 \mathrm{~Hz}$, F-phenyl-C), 156.9 (urea-C), 154.6 (purin-C), 152.9 (purin-C), 145.8 ( $t$-Bu-phenyl-C), 141.9 (purin-C), 139.0 (purin-C), 136.3 ( $t$-Bu-phenyl-C), 130.1 (d, J = 8.3 Hz, F-phenyl-C), 125.8 ( $t$-Bu-phenyl-C), 123.1 (d, J = 2.8 Hz, F-phenyl-C), 120.1 (purin-C), 119.5 ( $t$-Bu-phenyl-C), 114.5 (d, J = 21.6 Hz, F-phenyl-C), 114.2 (d, J = 20.8 Hz, F-phenyl-C), 89.8 (tetrahydrofuran-C), 
82.3 (tetrahydrofuran-C), 74.2 (tetrahydrofuran-C), 72.6 (tetrahydrofuran-C), $52.0\left(\mathrm{CH}_{2}-\mathrm{C}\right), 50.7$ $\left(\mathrm{CH}_{2}-\mathrm{C}\right), 48.5$ (isopropyl-CH-C), 43.8 (benzyl- $\left.\mathrm{CH}_{2}-\mathrm{C}\right), 38.2\left(\mathrm{CH}_{2}-\mathrm{C}\right), 34.2(t-\mathrm{Bu}-\mathrm{C}), 31.3$ ( $t$-Bu-CH${ }_{3}-\mathrm{C}$ ), $26.8\left(\mathrm{CH}_{2}-\mathrm{C}\right), 18.2$ (isopropyl- $\left.\mathrm{CH}_{3}-\mathrm{C}\right), 16.1$ (isopropyl- $\mathrm{CH}_{3}-\mathrm{C}$ ); LRMS (ESI): m/z $=671[\mathrm{M}+\mathrm{Na}]^{+}$; HRMS-ESI $(\mathrm{m} / \mathrm{z})$ : Calcd. For $\mathrm{C}_{34} \mathrm{H}_{46} \mathrm{~N}_{8} \mathrm{O}_{4} \mathrm{~F}(\mathrm{M}+\mathrm{H})^{+}:$649.3621; Found: 649.3611 .

4.2.13.5. 1-(3-((((2R,3S,4R,5R)-5-(6-((3-fluorobenzyl)amino)-9H-purin-9-yl)-3,4-dihydroxy tetrahydrofuran-2-yl)methyl)(isopropyl)amino)propyl)-3-(4-(trifluoromethyl)phenyl)urea 1ae

According to the general procedure, employing 8a and 1-isocyanato-4-(trifluoromethyl)benzene afforded compound 1ae as a solid, 85\% yield, HPLC purity: $95.8 \%$, method $\mathrm{B}$; mp: $119-120^{\circ} \mathrm{C} ;[\alpha]^{20}{ }_{\mathrm{D}}=10.73(\mathrm{c} 1.38, \mathrm{MeOH}) ;{ }^{1} \mathrm{H}$ NMR $(500 \mathrm{MHz}$, $\left.\mathrm{CDCl}_{3}\right) \delta 8.29(\mathrm{~s}, 1 \mathrm{H}$, purin-H), $8.19($ brs, $1 \mathrm{H}, \mathrm{NH}), 7.92(\mathrm{~s}, 1 \mathrm{H}$, purin- $\mathrm{H}), 7.45(\mathrm{~d}, \mathrm{~J}=8.1 \mathrm{~Hz}, 2 \mathrm{H}$, $\mathrm{CF}_{3}$-phenyl-H), $7.40\left(\mathrm{~d}, \mathrm{~J}=8.1 \mathrm{~Hz}, 2 \mathrm{H}, \mathrm{CF}_{3}\right.$-phenyl-H), 7.29-7.24 (m, $1 \mathrm{H}, \mathrm{F}-$ phenyl-H), 7.14 (d, $\mathrm{J}=7.5 \mathrm{~Hz}, 1 \mathrm{H}$, F-phenyl-H), $7.09(\mathrm{~d}, \mathrm{~J}=9.5 \mathrm{~Hz}, 1 \mathrm{H}, \mathrm{F}-$ phenyl-H), $6.96(\mathrm{t}, \mathrm{J}=7.5 \mathrm{~Hz}, 1 \mathrm{H}$, F-phenyl-H), 6.69 (brs, $2 \mathrm{H}, \mathrm{NH}$ and $\mathrm{NH}), 5.97$ (d, J = 3.3 Hz, 1H, tetrahydrofuran-H), 4.83 (brs, $2 \mathrm{H}$, benzyl- $\mathrm{CH}_{2}$ ), 4.64 (brs, $1 \mathrm{H}$, tetrahydrofuran- $\mathrm{H}$ ), 4.49 (brs, $1 \mathrm{H}$, tetrahydrofuran- $\mathrm{H}$ ), 4.35-4.32 (m, $1 \mathrm{H}$, tetrahydrofuran- $\mathrm{H}$ ), 3.27 (brs, $\left.2 \mathrm{H}, \mathrm{CH}_{2}\right), 3.14-3.10$ (m, $1 \mathrm{H}$, isopropyl- $\left.\mathrm{CH}\right), 2.97-2.83(\mathrm{~m}$, $\left.2 \mathrm{H}, \mathrm{CH}_{2}\right), 2.67$ (brs, $\left.2 \mathrm{H}, \mathrm{CH}_{2}\right), 1.71-1.68\left(\mathrm{~m}, 2 \mathrm{H}, \mathrm{CH}_{2}\right), 1.07\left(\mathrm{~d}, \mathrm{~J}=5.6 \mathrm{~Hz}, 3 \mathrm{H}\right.$, isopropyl- $\left.\mathrm{CH}_{3}\right)$, $0.98\left(\mathrm{~d}, \mathrm{~J}=5.6 \mathrm{~Hz}, 3 \mathrm{H}\right.$, isopropyl- $\left.\mathrm{CH}_{3}\right) ;{ }^{13} \mathrm{C} \mathrm{NMR}\left(400 \mathrm{MHz}, \mathrm{CDCl}_{3}\right) \delta 162.1(\mathrm{~d}, \mathrm{~J}=244.5 \mathrm{~Hz}$, F-phenyl-C), 156.1 (urea-C), 154.6 (purin-C), 152.9 (purin-C), 148.2 (F-phenyl-C), 142.6 ( $\mathrm{CF}_{3}$-phenyl-C), 140.9 (d, J = 6.9 Hz, F-phenyl-C), 138.6 (Ar-C), 130.1 (d, J = 8.2 Hz, Ar-C), 126.0 (d, J = 3.5 Hz, Ar-C), 124.2 (q, J = 272 Hz, $\left.\mathrm{CF}_{3}-\mathrm{C}\right), 123.5$ (q, J = 32.7 Hz, CF 3 -phenyl-C), 123.0 (d, J = 2.8 Hz, Ar-C), 120.04 (Ar-C), 114.5 (Ar-C), 114.2 (Ar-C), 89.8 (tetrahydrofuran-C), 82.3 (tetrahydrofuran-C), 74.3 (tetrahydrofuran-C), 72.8 (tetrahydrofuran-C), $52.0\left(\mathrm{CH}_{2}-\mathrm{C}\right), 51.3$ $\left(\mathrm{CH}_{2}-\mathrm{C}\right), 43.8$ (benzyl- $\left.\mathrm{CH}_{2}-\mathrm{C}\right), 38.4\left(\mathrm{CH}_{2}-\mathrm{C}\right), 26.3\left(\mathrm{CH}_{2}-\mathrm{C}\right), 18.1$ (isopropyl- $\left.\mathrm{CH}_{3}-\mathrm{C}\right), 16.0$ (isopropyl- $\mathrm{CH}_{3}-\mathrm{C}$ ); LRMS (ESI): $\mathrm{m} / \mathrm{z}=661[\mathrm{M}+\mathrm{H}]^{+}$; HRMS-ESI $(\mathrm{m} / \mathrm{z})$ : Calcd. For $\mathrm{C}_{31} \mathrm{H}_{37} \mathrm{~N}_{8} \mathrm{O}_{4} \mathrm{~F}_{4}(\mathrm{M}+\mathrm{H})^{+}$: 661.2868; Found: 661.2866 .

4.2.13.6. 1-(3-((((2R,3S,4R,5R)-5-(6-((3-fluorobenzyl)amino)-9H-purin-9-yl)-3,4-dihydroxy tetrahydrofuran-2-yl)methyl)(isopropyl)amino)propyl)-3-(3-(trifluoromethyl)phenyl)urea 1af

According to the general procedure, employing 8a and 1-isocyanato-3-(trifluoromethyl)benzene afforded compound 1af as an oil, 68\% yield, HPLC purity: $98.1 \%$, method $\mathrm{A} ;[\alpha]_{\mathrm{D}}^{20}=9.54(\mathrm{c} 0.71, \mathrm{MeOH}) ;{ }^{1} \mathrm{H}$ NMR $\left(500 \mathrm{MHz}, \mathrm{CDCl}_{3}\right) \delta 8.29(\mathrm{~s}$, $1 \mathrm{H}$, purin- $\mathrm{H}), 7.96(\mathrm{~s}, 1 \mathrm{H}, \mathrm{NH}), 7.78(\mathrm{~s}, 1 \mathrm{H}$, purin- $\mathrm{H}), 7.51\left(\mathrm{~d}, \mathrm{~J}=7.8 \mathrm{~Hz}, 1 \mathrm{H}, \mathrm{CF}_{3}\right.$-phenyl-H), 7.29-7.24 (m, 1H, Ar-H), 7.17-7.14 (m, 2H, Ar-H), 7.09 (d, J = 9.5 Hz, 1H, Ar-H), 6.96 (t, J = 7.5 $\mathrm{Hz}, 1 \mathrm{H}, \mathrm{Ar}-\mathrm{H}), 6.75$ (brs, $1 \mathrm{H}, \mathrm{NH}), 6.50$ (brs, $1 \mathrm{H}, \mathrm{NH}), 5.99(\mathrm{~d}, \mathrm{~J}=3.3 \mathrm{~Hz}, 1 \mathrm{H}$, tetrahydrofuran- $\mathrm{H}$ ), 4.83 (brs, $2 \mathrm{H}$, benzyl- $\left.\mathrm{CH}_{2}\right), 4.65$ (brs, $2 \mathrm{H}$, tetrahydrofuran- $\left.\mathrm{H}\right), 4.51$ (brs, $1 \mathrm{H}$, tetrahydrofuran- $\mathrm{H}), 3.32-2.87\left(\mathrm{~m}, 7 \mathrm{H}, 3 \times \mathrm{CH}_{2}\right.$ and isopropyl- $\left.\mathrm{CH}\right), 1.87\left(\mathrm{brs}, 2 \mathrm{H}, \mathrm{CH}_{2}\right), 1.19(\mathrm{~d}, \mathrm{~J}$ $=5.6 \mathrm{~Hz}, 3 \mathrm{H}$, isopropyl- $\left.\mathrm{CH}_{3}\right), 1.07\left(\mathrm{~d}, \mathrm{~J}=5.6 \mathrm{~Hz}, 3 \mathrm{H}\right.$, isopropyl- $\left.\mathrm{CH}_{3}\right) ;{ }^{13} \mathrm{C} \mathrm{NMR}(400 \mathrm{MHz}$, $\left.\mathrm{CDCl}_{3}\right) \delta 162.1(\mathrm{~d}, \mathrm{~J}=244.5 \mathrm{~Hz}, \mathrm{~F}-$ phenyl-C), 156.1 (urea-C), 154.6 (purin-C), 152.9 (purin-C), 141.0 (purin-C), $140.0\left(\mathrm{CF}_{3}\right.$-phenyl-C), 139.2 (purin-C), 131.0 (q, $\mathrm{J}=32.5 \mathrm{~Hz}, \mathrm{CF}_{3}$-phenyl-C), 130.2 (d, J = 8.4 Hz, F-phenyl-C), 129.2 (Ar-C), 123.2 (q, J = 272.1 Hz, $\mathrm{CF}_{3}-\mathrm{C}$ ), 123.1 (Ar-C), 121.6 (Ar-C), 118.6 (Ar-C), 115.0 (Ar-C), 114.5 (d, J = 21.7 Hz, F-phenyl-C), 114.3 (d, J = 21.5 $\mathrm{Hz}, \mathrm{F}$-phenyl-C), 90.2 (tetrahydrofuran-C), 82.3 (tetrahydrofuran-C), 74.1 (tetrahydrofuran-C), 72.5 (tetrahydrofuran-C), $52.0\left(\mathrm{CH}_{2}-\mathrm{C}\right), 48.9\left(\mathrm{CH}_{2}-\mathrm{C}\right), 43.2$ (benzyl- $\left.\mathrm{CH}_{2}-\mathrm{C}\right), 37.6\left(\mathrm{CH}_{2}-\mathrm{C}\right), 26.0$ $\left(\mathrm{CH}_{2}-\mathrm{C}\right.$ ), 17.7 (isopropyl- $\mathrm{CH}_{3}-\mathrm{C}$ ), 15.9 (isopropyl- $\mathrm{CH}_{3}-\mathrm{C}$ ); LRMS (ESI): $\mathrm{m} / \mathrm{z}=661[\mathrm{M}+\mathrm{Na}]^{+}$; HRMS-ESI (m/z): Calcd. For $\mathrm{C}_{31} \mathrm{H}_{37} \mathrm{~N}_{8} \mathrm{O}_{4} \mathrm{~F}_{4}(\mathrm{M}+\mathrm{H})^{+}$: 661.2868; Found: 661.2867. 
4.2.13.7.

1-(tert-butyl)-3-(3-((((2R,3S,4R,5R)-5-(6-((3-fluorobenzyl)amino)-9H-purin-9-yl)-3,4-dihydroxyt etrahydrofuran-2-yl)methyl)(isopropyl)amino)propyl)urea $\mathbf{1 a g}$

According to the general procedure, employing 8a and 2-isocyanato-2-methylpropane afforded compound 1ag as an oil, 73\% yield, HPLC purity: 97.2\%, method $\mathrm{A} ;[\alpha]_{\mathrm{D}}^{20}=1.43$ (c 0.77 , $\mathrm{MeOH}) ;{ }^{1} \mathrm{H}$ NMR $\left(500 \mathrm{MHz}, \mathrm{CDCl}_{3}\right) \delta 8.37(\mathrm{~s}, 1 \mathrm{H}$, purin-H), $7.97(\mathrm{~s}, 1 \mathrm{H}$, purin-H), 7.29-7.24 (m, $1 \mathrm{H}, \mathrm{Ar}-\mathrm{H}), 7.14(\mathrm{~d}, \mathrm{~J}=7.5 \mathrm{~Hz}, 1 \mathrm{H}, \mathrm{Ar}-\mathrm{H}), 7.09(\mathrm{~d}, \mathrm{~J}=9.5 \mathrm{~Hz}, 1 \mathrm{H}, \mathrm{Ar}-\mathrm{H}), 6.96(\mathrm{t}, \mathrm{J}=7.5 \mathrm{~Hz}, 1 \mathrm{H}$, Ar-H), 6.52 (brs, $1 \mathrm{H}, \mathrm{NH}), 5.99(\mathrm{~d}, \mathrm{~J}=3.3 \mathrm{~Hz}, 1 \mathrm{H}$, tetrahydrofuran-H), 4.89 (brs, $2 \mathrm{H}$, benzyl- $\left.\mathrm{CH}_{2}\right), 4.74$ (brs, $1 \mathrm{H}$, tetrahydrofuran- $\left.\mathrm{H}\right), 4.60$ (brs, $1 \mathrm{H}$, tetrahydrofuran- $\left.\mathrm{H}\right), 4.35-4.32(\mathrm{~m}$, $1 \mathrm{H}$, tetrahydrofuran- $\mathrm{H}$ ), 3.27 (brs, $\left.2 \mathrm{H}, \mathrm{CH}_{2}\right), 3.14-3.10(\mathrm{~m}, 1 \mathrm{H}$, isopropyl- $\mathrm{CH}), 3.04-2.86(\mathrm{~m}, 2 \mathrm{H}$, $\left.\mathrm{CH}_{2}\right), 2.72$ (brs, $\left.2 \mathrm{H}, \mathrm{CH}_{2}\right), 1.75-1.72\left(\mathrm{~m}, 2 \mathrm{H}, \mathrm{CH}_{2}\right), 1.32(\mathrm{~s}, 9 \mathrm{H}, t-\mathrm{Bu}), 1.16(\mathrm{~d}, \mathrm{~J}=5.6 \mathrm{~Hz}, 3 \mathrm{H}$, isopropyl- $\left.\mathrm{CH}_{3}\right), 1.03\left(\mathrm{~d}, \mathrm{~J}=5.6 \mathrm{~Hz}, 3 \mathrm{H}\right.$, isopropyl- $\left.\mathrm{CH}_{3}\right) ;{ }^{13} \mathrm{C} \mathrm{NMR}\left(400 \mathrm{MHz}, \mathrm{CDCl}_{3}\right) \delta 162.1(\mathrm{~d}$, $\mathrm{J}=244.5 \mathrm{~Hz}, \mathrm{~F}-$ phenyl-C), 158.8 (urea-C), 154.6 (purin-C), 152.9 (purin-C), 148.6 (purin-C), 141.2 (d, J = 6.9 Hz, F-phenyl-C), 139.2 (purin-C), 130.2 (d, J = 8.2 Hz, F-phenyl-C), 123.0 (d, J $=2.8 \mathrm{~Hz}$, F-phenyl-C), 120.3 (purin-C), $114.5(\mathrm{~d}, \mathrm{~J}=21.7 \mathrm{~Hz}, \mathrm{~F}-$ phenyl-C), $114.3(\mathrm{~d}, \mathrm{~J}=21.8 \mathrm{~Hz}$, F-phenyl-C), 90.0 (tetrahydrofuran-C), 82.6 (tetrahydrofuran-C), 74.0 (tetrahydrofuran-C), 72.3 (tetrahydrofuran-C), $51.8\left(\mathrm{CH}_{2}-\mathrm{C}\right), 50.3(\mathrm{t}-\mathrm{Bu}-\mathrm{C}), 48.6\left(\mathrm{CH}_{2}-\mathrm{C}\right), 43.8$ (benzyl- $\left.\mathrm{CH}_{2}-\mathrm{C}\right), 38.6$ $\left(\mathrm{CH}_{2}-\mathrm{C}\right), 29.7\left(\mathrm{t}-\mathrm{Bu}-\mathrm{CH}_{3}-\mathrm{C}\right), 26.7\left(\mathrm{CH}_{2}-\mathrm{C}\right), 18.6$ (isopropyl- $\left.\mathrm{CH}_{3}-\mathrm{C}\right), 15.8$ (isopropyl- $\mathrm{CH}_{3}-\mathrm{C}$ ); LRMS (ESI): $\mathrm{m} / \mathrm{z}=573[\mathrm{M}+\mathrm{H}]^{+}$; HRMS-ESI (m/z): Calcd. For $\mathrm{C}_{28} \mathrm{H}_{42} \mathrm{~N}_{8} \mathrm{O}_{4} \mathrm{~F}(\mathrm{M}+\mathrm{H})^{+}: 573.3308$; Found: 573.3317.

\subsubsection{8.}

1-cyclohexyl-3-(3-((((2R,3S,4R,5R)-5-(6-((3-fluorobenzyl)amino)-9H-purin-9-yl)-3,4-dihydroxyt etrahydrofuran-2-yl)methyl)(isopropyl)amino)propyl)urea 1ah

According to the general procedure, employing 8a and 1-isocyanato-4-methoxylbenzene afforded compound 1ah as an oil, 76\% yield, HPLC purity: $96.8 \%$, method $\mathrm{B} ;[\alpha]_{\mathrm{D}}^{20}=5.05(\mathrm{c}$ $0.69, \mathrm{MeOH}) ;{ }^{1} \mathrm{H}$ NMR $(500 \mathrm{MHz}, \mathrm{MeOD}) \delta 8.31(\mathrm{~s}, 1 \mathrm{H}$, purin-H), $8.25(\mathrm{~s}, 1 \mathrm{H}$, purin- $\mathrm{H})$, 7.40-7.34 (m, 1H, Ar-H), 7.20 (d, J = 7.5 Hz, 1H, Ar-H), $7.15(\mathrm{~d}, \mathrm{~J}=9.5 \mathrm{~Hz}, 1 \mathrm{H}, \mathrm{Ar}-\mathrm{H}), 7.00$ (t, J $=7.5 \mathrm{~Hz}, 1 \mathrm{H}, \mathrm{Ar}-\mathrm{H}), 6.04\left(\mathrm{~d}, \mathrm{~J}=4.0 \mathrm{~Hz}, 1 \mathrm{H}\right.$, tetrahydrofuran-H), 4.88 (brs, $2 \mathrm{H}$, benzyl- $\mathrm{CH}_{2}$ ), 4.79 (brs, $1 \mathrm{H}$, tetrahydrofuran- $\mathrm{H}), 4.37$ (brs, $1 \mathrm{H}$, tetrahydrofuran- $\mathrm{H}), 4.23$ (brs, $2 \mathrm{H}$, etrahydrofuran-H), 0.98 (brs, $1 \mathrm{H}$, isopropyl-CH), 3.46-3.43 (m, 1H, cyclohexyl-H), 3.24-2.72 (m, $7 \mathrm{H}$, cyclohexyl-CH-H and $\left.3 \times \mathrm{CH}_{2}\right), 1.85-1.61\left(\mathrm{~m}, 8 \mathrm{H}, \mathrm{CH}_{2}\right.$ and cyclohexyl-6H), 1.23-1.10 (m, $10 \mathrm{H}, 2 \times \mathrm{CH}_{3}$ and cyclohexyl-4H); ${ }^{13} \mathrm{C}$ NMR $(400 \mathrm{MHz}, \mathrm{MeOD}) \delta 162.1(\mathrm{~d}, \mathrm{~J}=244.5 \mathrm{~Hz}, \mathrm{Ar}-\mathrm{H})$, 159.1 (urea-C), 154.6 (purin-C), 152.5 (purin-C), 142.0 (Ar-C), 139.8 (purin-C), 129.7 (d, J = 8.2 $\mathrm{Hz}$, Ar-C), 122.7 (d, J = 2.8 Hz, Ar-C), 119.7 (purin-C), 113.5 (d, J = 22.2 Hz, Ar-C), 113.3 (d, J $=22.4 \mathrm{~Hz}, \mathrm{Ar}-\mathrm{C}$ ), 89.2 (tetrahydrofuran-C), 82.7 (tetrahydrofuran-C), 73.1 (tetrahydrofuran-C), 72.0 (tetrahydrofuran-C), $52.0\left(\mathrm{CH}_{2}-\mathrm{C}\right), 48.5\left(\mathrm{CH}_{2}-\mathrm{C}\right), 43.2$ (benzyl- $\left.\mathrm{CH}_{2}-\mathrm{C}\right), 37.5\left(\mathrm{CH}_{2}-\mathrm{C}\right), 33.2$ (cyclohexyl- $\left.\mathrm{CH}_{2}-\mathrm{C}\right), 26.6\left(\mathrm{CH}_{2}-\mathrm{C}\right), 25.4$ (cyclohexyl- $\left.\mathrm{CH}_{2}-\mathrm{C}\right), 25.2\left(\right.$ cyclohexyl- $\left.\mathrm{CH}_{2}-\mathrm{C}\right), 16.7$ (isopropyl- $\mathrm{CH}_{3}-\mathrm{C}$ ), 15.8 (isopropyl- $\mathrm{CH}_{3}-\mathrm{C}$ ); LRMS (ESI): $\mathrm{m} / \mathrm{z}=599[\mathrm{M}+\mathrm{Na}]^{+}$; HRMS-ESI (m/z): Calcd. For $\mathrm{C}_{30} \mathrm{H}_{44} \mathrm{~N}_{8} \mathrm{O}_{4} \mathrm{~F}_{4}(\mathrm{M}+\mathrm{H})^{+}$: 599.3464; Found: 599.3464 .

4.2.13.9.

1-(3-((((2R,3S,4R,5R)-5-(6-((3-chlorobenzyl)amino)-9H-purin-9-yl)-3,4-dihydroxytetrahydrofura n-2-yl)methyl)(isopropyl)amino)propyl)-3-(4-fluorophenyl)urea 1ba

According to the general procedure, employing $\mathbf{8 b}$ and 1-isocyanato-4-methoxylbenzene afforded compound $\mathbf{1 b a}$ as a solid, $75 \%$ yield, HPLC purity: $99.6 \%$, method A; mp: $189-191^{\circ} \mathrm{C}$; $[\alpha]^{20}{ }_{\mathrm{D}}=-25.34$ (c 0.23, DMSO); ${ }^{1} \mathrm{H}$ NMR $\left(500 \mathrm{MHz}, \mathrm{DMSO}-d_{6}\right) \delta 8.47$ (brs, $1 \mathrm{H}$, purin-H), 8.43 (s, $1 \mathrm{H}, \mathrm{NH}), 8.22(\mathrm{~s}, 1 \mathrm{H}$, purin-H), 7.39-7.35 (m, 3H, Ar-H), 7.33-7.25 (m, 3H, Ar-H), 7.06-7.01 $(\mathrm{m}, 2 \mathrm{H}, \mathrm{Ar}-\mathrm{H}), 6.13$ (brs, $1 \mathrm{H}, \mathrm{NH}), 5.88(\mathrm{~d}, \mathrm{~J}=6.9 \mathrm{~Hz}, 1 \mathrm{H}$, tetrahydrofuran-H), $5.44(\mathrm{~d}, \mathrm{~J}=7.4$ 
$\mathrm{Hz}, 1 \mathrm{H}$, tetrahydrofuran- $\mathrm{H}), 5.18(\mathrm{~d}, \mathrm{~J}=5.8 \mathrm{~Hz}, 1 \mathrm{H}$, tetrahydrofuran- $\mathrm{H}), 4.76-4.70(\mathrm{~m}, 3 \mathrm{H}$, tetrahydrofuran- $\mathrm{H}$ and benzyl- $\mathrm{CH}_{2}$ ), 4.15 (brs, $1 \mathrm{H}, \mathrm{CH}_{2}-1 \mathrm{H}$ ), 3.93 (brs, $1 \mathrm{H}, \mathrm{CH}_{2}-1 \mathrm{H}$ ), 3.08-3.06 (m, 2H, $\left.\mathrm{CH}_{2}\right), 2.98-2.92(\mathrm{~m}, 1 \mathrm{H}$, isopropyl-CH), 2.86-2.78 (m, 1H, $\mathrm{NH}), 2.46-2.40\left(\mathrm{~m}, 2 \mathrm{H}, \mathrm{CH}_{2}\right)$, 1.54-1.50 (m, 2H, $\left.\mathrm{CH}_{2}\right), 0.97\left(\mathrm{~d}, \mathrm{~J}=6.3 \mathrm{~Hz}, 3 \mathrm{H}\right.$, isopropyl- $\left.\mathrm{CH}_{3}\right), 0.88(\mathrm{~d}, \mathrm{~J}=6.3 \mathrm{~Hz}, 3 \mathrm{H}$, isopropyl- $\left.\mathrm{CH}_{3}\right) ;{ }^{13} \mathrm{C}$ NMR (400 MHz, DMSO- $\left.d_{6}\right) \delta 157.2(\mathrm{~d}, \mathrm{~J}=237.5 \mathrm{~Hz}$, F-phenyl-C), 155.7 (urea-C), 154.7 (purin-C), 152.9 (purin-C), 148.3 (Cl-phenyl-C), 143.2 (purin-C), 140.6 (purin-C), 137.4 (F-phenyl-C), 133.3 (Ar-C), 130.5 (Ar-C), 127.3 (Ar-C), 127.0 (Ar-C), 126.2 (Ar-C), 119.6 $(\mathrm{d}, \mathrm{J}=7.4 \mathrm{~Hz}, \mathrm{~F}-$ phenyl-C), 115.6 (Ar-C), 115.3 (Ar-C), 87.8 (tetrahydrofuran-C), 84.3 (tetrahydrofuran-C), 72.8 (tetrahydrofuran-C), 72.1 (tetrahydrofuran-C), $52.6\left(\mathrm{CH}_{2}-\mathrm{C}\right), 50.7$ $\left(\mathrm{CH}_{2}-\mathrm{C}\right), 48.1$ (isopropyl-CH-C), 42.8 (benzyl- $\left.\mathrm{CH}_{2}-\mathrm{C}\right), 37.8\left(\mathrm{CH}_{2}-\mathrm{C}\right), 29.4\left(\mathrm{CH}_{2}-\mathrm{C}\right), 19.4$ (isopropyl- $\mathrm{CH}_{3}-\mathrm{C}$ ), 17.1 (isopropyl- $\mathrm{CH}_{3}-\mathrm{C}$ ); LRMS (ESI): $\mathrm{m} / \mathrm{z}=627[\mathrm{M}+\mathrm{H}]^{+}$; HRMS-ESI $(\mathrm{m} / \mathrm{z})$ : Calcd. For $\mathrm{C}_{30} \mathrm{H}_{37} \mathrm{~N}_{8} \mathrm{O}_{4} \mathrm{FCl}(\mathrm{M}+\mathrm{H})^{+}$: 627.2605; Found: 627.2622 .

\subsubsection{0.}

1-(3-((( (2R,3S,4R,5R)-5-(6-((3-chlorobenzyl)amino)-9H-purin-9-yl)-3,4-dihydroxytetrahydrofura n-2-yl)methyl)(isopropyl)amino)propyl)-3-(p-tolyl)urea $\mathbf{1 b b}$

According to the general procedure, employing $8 \mathbf{b}$ and 1-isocyanato-4-methylbenzene afforded compound 1bb as a solid, 65\% yield, HPLC purity: $92.0 \%$, method $\mathrm{A} ; \mathrm{mp}: 180-181^{\circ} \mathrm{C} ;[\alpha]^{20}{ }_{\mathrm{D}}=$ 13.69 (c $0.45, \mathrm{MeOH}) ;{ }^{1} \mathrm{H}$ NMR $(500 \mathrm{MHz}, \mathrm{MeOD}) \delta 8.30$ (s, $1 \mathrm{H}$, purin-H), $8.18(\mathrm{~s}, 1 \mathrm{H}$, purin-H), 7.41 (s, 1H, Ar-H), 7.33-7.28 (m, 3H, Ar-H), 7.20 (d, J = 8.8 Hz, 2H, Me-phenyl-H), $7.05(\mathrm{~d}, \mathrm{~J}=8.8 \mathrm{~Hz}, 2 \mathrm{H}$, Me-phenyl-H), $6.01(\mathrm{~d}, \mathrm{~J}=4.0 \mathrm{~Hz}, 1 \mathrm{H}$, tetrahydrofuran-H), 4.79 (brs, $2 \mathrm{H}$, benzyl- $\left.\mathrm{CH}_{2}\right), 4.78-4.76(\mathrm{~m}, 1 \mathrm{H}$, tetrahydrofuran- $\mathrm{H}), 4.49$ (brs, $1 \mathrm{H}$, tetrahydrofuran- $\left.\mathrm{H}\right), 4.40$ (brs, $1 \mathrm{H}$, tetrahydrofuran- $\mathrm{H}), 3.38-3.35\left(\mathrm{~m}, 3 \mathrm{H}, \mathrm{CH}_{2}\right.$ and isopropyl- $\left.\mathrm{CH}\right), 3.28-3.17\left(\mathrm{~m}, 4 \mathrm{H}, 2 \times \mathrm{CH}_{2}\right)$, 2.28 (s, 3H, tolyl- $\left.\mathrm{CH}_{3}\right), 1.92$ (brs, $\left.2 \mathrm{H}, \mathrm{CH}_{2}\right), 1.29$ (s, 3H, isopropyl- $\left.\mathrm{CH}_{3}\right), 0.94(\mathrm{~s}, 3 \mathrm{H}$, isopropyl- $\mathrm{CH}_{3}$ ); ${ }^{13} \mathrm{C}$ NMR (400 MHz, MeOD) $\delta 157.4$ (urea-C), 152.5 (purin-C), 141.5 (Ar-C), 140.0 (purin-C), 136.4 (purin-C), 133.9 (Ar-C), 131.9 (Ar-C), 129.5 (Ar-C), 129.3 (Ar-C), 128.8 (Ar-C), 128.4 (Ar-C), 126.9 (Ar-C), 126.7 (Ar-C), 125.3 (Ar-C), 119.2 (Ar-C), 90.2 (tetrahydrofuran-C), 80.2 (tetrahydrofuran-C), 72.9 (tetrahydrofuran-C), 72.1 (tetrahydrofuran-C), $51.9\left(\mathrm{CH}_{2}-\mathrm{C}\right), 48.6\left(\mathrm{CH}_{2}-\mathrm{C}\right), 42.9$ (benzyl-C), $26.6\left(\mathrm{CH}_{2}-\mathrm{C}\right), 22.2$ (isopropyl- $\left.\mathrm{CH}_{3}-\mathrm{C}\right), 19.3$ (isopropyl- $\mathrm{CH}_{3}-\mathrm{C}$ ); LRMS (ESI): $\mathrm{m} / \mathrm{z}=623[\mathrm{M}+\mathrm{H}]^{+}$; HRMS-ESI $(\mathrm{m} / \mathrm{z}$ ): Calcd. For $\mathrm{C}_{31} \mathrm{H}_{40} \mathrm{~N}_{8} \mathrm{O}_{4} \mathrm{Cl}(\mathrm{M}+\mathrm{H})^{+}$: 623.2856; Found: 623.2858 .

4.2.13.11. 1-(3-((( $\quad$ - $2 \mathrm{R}, 3 \mathrm{~S}, 4 \mathrm{R}, 5 \mathrm{R})-5-(6-((3-$ chlorobenzyl)amino)-9H-purin-9-yl)-3,4-dihydroxy tetrahydrofuran-2-yl)methyl)(isopropyl)amino)propyl)-3-(4-methoxyphenyl)urea 1bc

According to the general procedure, employing $\mathbf{8 b}$ and 1-isocyanato-4-methoxylbenzene afforded compound $1 \mathrm{bc}$ as a solid, $77 \%$ yield, HPLC purity: $93.6 \%$, method A; mp: $170-171^{\circ} \mathrm{C}$; $[\alpha]^{20}{ }_{\mathrm{D}}=10.88(\mathrm{c} 0.38, \mathrm{MeOH}) ;{ }^{1} \mathrm{H}$ NMR $(500 \mathrm{MHz}, \mathrm{MeOD}) \delta 8.29(\mathrm{~s}, 1 \mathrm{H}$, purin- $\mathrm{H}), 8.22(\mathrm{~s}, 1 \mathrm{H}$, purin-H), 7.41 (s, 1H, Ar-H), 7.33-7.28 (m, 3H, Ar-H), 7.20 (d, J = 8.8 Hz, 2H, MeO-phenyl-H), $6.84(\mathrm{~d}, \mathrm{~J}=8.8 \mathrm{~Hz}, 2 \mathrm{H}, \mathrm{MeO}-$ phenyl-H), $6.02(\mathrm{~d}, \mathrm{~J}=4.0 \mathrm{~Hz}, 1 \mathrm{H}$, tetrahydrofuran-H), 4.79 (brs, $2 \mathrm{H}$, benzyl- $\left.\mathrm{CH}_{2}\right), 4.78-4.76(\mathrm{~m}, 1 \mathrm{H}$, tetrahydrofuran- $\mathrm{H}), 4.49$ (brs, $1 \mathrm{H}$, tetrahydrofuran- $\left.\mathrm{H}\right), 4.34$ (brs, $1 \mathrm{H}$, tetrahydrofuran- $\mathrm{H}), 3.79\left(\mathrm{~s}, 3 \mathrm{H}, \mathrm{MeO}-\mathrm{CH}_{3}\right), 3.38-3.35\left(\mathrm{~m}, 3 \mathrm{H}, \mathrm{CH}_{2}\right.$ and isopropyl-CH), 3.28-3.17 (m, 4H, $\left.2 \times \mathrm{CH}_{2}\right), 1.84$ (brs, $\left.2 \mathrm{H}, \mathrm{CH}_{2}\right), 1.29$ (s, 3H, isopropyl- $\left.\mathrm{CH}_{3}\right), 0.94(\mathrm{~s}, 3 \mathrm{H}$, isopropyl- $\mathrm{CH}_{3}$ ); ${ }^{13} \mathrm{C}$ NMR (400 MHz, MeOD) 157.7 (Meo-phenyl-C), 155.7 (urea-C), 152.5 (purin-C), 141.5 (purin-C), 139.9 (purin-C), 131.8 (Cl-phenyl-C), 129.5 (Cl-phenyl-C), 129.3 (purin-C), 126.9 (MeO-phenyl-C), 126.7 (MeO-phenyl-C), 125.3 (Cl-phenyl-C), 121.4 (Cl-phenyl-C), 113.6 (MeO-phenyl-C), 89.9 (tetrahydrofuran-C), 73.0 (tetrahydrofuran-C), 72.1 (tetrahydrofuran-C), $54.4\left(\mathrm{CH}_{2}-\mathrm{C}\right), 51.9\left(\mathrm{CH}_{2}-\mathrm{C}\right), 48.5$ (isopropyl-CH-C), 42.9 (benzyl- $\mathrm{CH}_{2}-\mathrm{C}$ ), $31.6\left(\mathrm{CH}_{2}-\mathrm{C}\right.$ ), $26.6\left(\mathrm{CH}_{2}-\mathrm{C}\right.$ ), 25.4 (isopropyl- $\mathrm{CH}_{3}-\mathrm{C}$ ), 22.3 (isopropyl- $\mathrm{CH}_{3}-\mathrm{C}$ ); LRMS (ESI): m/z 
$=639[\mathrm{M}+\mathrm{H}]^{+}$; HRMS-ESI $(\mathrm{m} / \mathrm{z})$ : Calcd. For $\mathrm{C}_{31} \mathrm{H}_{40} \mathrm{~N}_{8} \mathrm{O}_{5} \mathrm{Cl}(\mathrm{M}+\mathrm{H})^{+}$: 639.2805; Found: 639.2812 .

\subsubsection{2.}

1-(4-(tert-butyl)phenyl)-3-(3-((((2R,3S,4R,5R)-5-(6-((3-chlorobenzyl)amino)-9H-purin-9-yl)-3,4dihydroxytetrahydrofuran-2-yl)methyl)(isopropyl)amino)propyl)urea $\mathbf{1 b d}$

According to the general procedure, employing $8 \mathbf{b}$ and 1-isocyanato-4-methoxylbenzene afforded compound 1bd as a solid, 73\% yield, HPLC purity: $99.3 \%$, method A; mp: $122-124^{\circ} \mathrm{C}$; $[\alpha]^{20}{ }_{\mathrm{D}}=12.72(\mathrm{c} 0.78, \mathrm{MeOH}) ;{ }^{1} \mathrm{H}$ NMR $(500 \mathrm{MHz}, \mathrm{MeOD}) \delta 8.27(\mathrm{~s}, 1 \mathrm{H}$, purin- $\mathrm{H}), 8.25(\mathrm{~s}, 1 \mathrm{H}$, purin-H), $7.41(\mathrm{~s}, 1 \mathrm{H}, \mathrm{Cl}$-phenyl-H), 7.32-7.22 (m, 7H, Ar-H), $6.02(\mathrm{~d}, \mathrm{~J}=4.0 \mathrm{~Hz}, 1 \mathrm{H}$, tetrahydrofuran- $\mathrm{H}), 4.8$ (brs, $2 \mathrm{H}$, benzyl- $\left.\mathrm{CH}_{2}\right), 4.79-4.76(\mathrm{~m}, 1 \mathrm{H}$, tetrahydrofuran- $\mathrm{H}), 4.35-4.32$ $(\mathrm{m}, 1 \mathrm{H}$, tetrahydrofuran- $\mathrm{H}), 4.22-4.18(\mathrm{~m}, 1 \mathrm{H}$, tetrahydrofuran- $\mathrm{H}), 3.29-3.20\left(\mathrm{~m}, 2 \mathrm{H}, \mathrm{CH}_{2}\right), 3.09$ (brs, $1 \mathrm{H}$, isopropyl- $\mathrm{CH}), 2.96\left(\mathrm{~d}, \mathrm{~J}=12.9 \mathrm{~Hz}, 1 \mathrm{H}, \mathrm{CH}_{2}-1 \mathrm{H}\right), 2.79$ (brs, $\left.1 \mathrm{H}, \mathrm{CH}_{2}-1 \mathrm{H}\right), 2.62$ (brs, $\left.2 \mathrm{H}, \mathrm{CH}_{2}\right), 1.70-1.68\left(\mathrm{~m}, 2 \mathrm{H}, \mathrm{CH}_{2}\right), 1.07\left(\mathrm{~d}, \mathrm{~J}=5.7 \mathrm{~Hz}, 3 \mathrm{H}\right.$, isopropyl- $\left.\mathrm{CH}_{3}\right), 1.02(\mathrm{~d}, \mathrm{~J}=5.7 \mathrm{~Hz}$, $3 \mathrm{H}$, isopropyl- $\mathrm{CH}_{3}$ ); ${ }^{13} \mathrm{C}$ NMR (400 MHz, MeOD) 157.0 (urea-C), 152.4 (purin-C), 144.9 (Cl-phenyl-C), 141.4 (purin-C), 139.6 (purin-C), 136.6 ( $t$-Bu-phenyl-C), 133.9 (Cl-phenyl-C), 129.5 (Cl-phenyl-C), 126.9 (Cl-phenyl-C), 126.7 (Cl-phenyl-C), 125.3 (Cl-phenyl-C), 125.0 ( $t$-Bu-phenyl-C), 118.8 ( $t$-Bu-phenyl-C), 89.0 (tetrahydrofuran-C), 83.2 (tetrahydrofuran-C), 73.2 (tetrahydrofuran-C), 72.0 (tetrahydrofuran-C), $52.1\left(\mathrm{CH}_{2}-\mathrm{C}\right), 50.6\left(\mathrm{CH}_{2}-\mathrm{C}\right), 42.9$ (benzyl- $\mathrm{CH}_{2}-\mathrm{C}$ ), $39.0\left(\mathrm{CH}_{2}-\mathrm{C}\right), 30.4\left(t-\mathrm{Bu}_{-} \mathrm{CH}_{3}-\mathrm{C}\right), 27.8\left(\mathrm{CH}_{2}-\mathrm{C}\right), 17.0$ (isopropyl- $\left.\mathrm{CH}_{3}-\mathrm{C}\right), 16.2$ (isopropyl- $\left.\mathrm{CH}_{3}-\mathrm{C}\right)$; LRMS (ESI): $\mathrm{m} / \mathrm{z}=687[\mathrm{M}+\mathrm{Na}]^{+}$; HRMS-ESI $(\mathrm{m} / \mathrm{z}):$ Calcd. For $\mathrm{C}_{34} \mathrm{H}_{46} \mathrm{~N}_{8} \mathrm{O}_{4} \mathrm{Cl}(\mathrm{M}+\mathrm{H})^{+}$: 665.3325; Found: 665.3339.

4.2.13.13. 1-(3-((((2R,3S,4R,5R)-5-(6-((3-chlorobenzyl)amino)-9H-purin-9-yl)-3,4-dihydroxy tetrahydrofuran-2-yl)methyl)(isopropyl)amino)propyl)-3-(4-(trifluoromethyl)phenyl)urea 1be

According to the general procedure, employing $\mathbf{8 b}$ and 1-isocyanato-4-methoxylbenzene afforded compound 1 be as a solid, $80 \%$ yield, HPLC purity: $97.6 \%$, method $\mathrm{B}$; mp: $116-117^{\circ} \mathrm{C}$; $[\alpha]^{20}{ }_{\mathrm{D}}=11.53(\mathrm{c} 0.76, \mathrm{MeOH}) ;{ }^{1} \mathrm{H}$ NMR $(500 \mathrm{MHz}, \mathrm{MeOD}) \delta 8.25$ (s, 2H, purin-H), 7.52-7.48 (m, 4H, CF - phenyl-H), 7.41 (s, 1H, Cl-phenyl-H), 7.33-7.27 (m, 3H, Cl-phenyl-H), 6.02 (d, J = $4.0 \mathrm{~Hz}, \quad 1 \mathrm{H}$, tetrahydrofuran- $\mathrm{H}), 4.88$ (brs, $2 \mathrm{H}$, benzyl- $\left.\mathrm{CH}_{2}\right), \quad 4.79-4.76(\mathrm{~m}, 1 \mathrm{H}$, tetrahydrofuran- $\mathrm{H}), 4.35-4.32(\mathrm{~m}, 1 \mathrm{H}$, tetrahydrofuran- $\mathrm{H}), 4.22-4.18(\mathrm{~m}, 1 \mathrm{H}$, tetrahydrofuran- $\mathrm{H})$, 3.30-3.20 (m, 2H, $\left.\mathrm{CH}_{2}\right), 3.09$ (brs, $1 \mathrm{H}$, isopropyl-CH), $2.96\left(\mathrm{~d}, \mathrm{~J}=12.9 \mathrm{~Hz}, 1 \mathrm{H}, \mathrm{CH}_{2}-1 \mathrm{H}\right), 2.79$ (brs, $1 \mathrm{H}, \mathrm{CH}_{2}-1 \mathrm{H}$ ), 2.62 (brs, $\left.2 \mathrm{H}, \mathrm{CH}_{2}\right), 1.70-1.68\left(\mathrm{~m}, 2 \mathrm{H}, \mathrm{CH}_{2}\right), 1.08(\mathrm{~d}, \mathrm{~J}=5.7 \mathrm{~Hz}, 3 \mathrm{H}$, isopropyl- $\left.\mathrm{CH}_{3}\right), 1.04\left(\mathrm{~d}, \mathrm{~J}=5.7 \mathrm{~Hz}, 3 \mathrm{H}\right.$, isopropyl- $\left.\mathrm{CH}_{3}\right) ;{ }^{13} \mathrm{C}$ NMR $(400 \mathrm{MHz}, \mathrm{MeOD}) 156.1$ (urea-C), 154.5 (purin-C), 152.4 (purin-C), 148.5 (Cl-phenyl-C), 143.3 (purin-C), 141.4 (purin-C), 139.5 ( $\mathrm{CF}_{3}$-phenyl-C), 133.9 (Cl-phenyl-C), 129.5 (Cl-phenyl-C), 126.9 (Cl-phenyl-C), 126.7

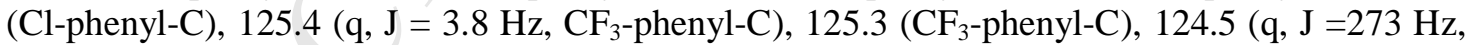
$\mathrm{CF}_{3}-\mathrm{C}$ ), 12.9 (q, J = 32.3 Hz, $\mathrm{CF}_{3}$-phenyl-C), 119.6 (purin-C), $117.5\left(\mathrm{CF}_{3}\right.$-phenyl-C), 89.0 (tetrahydrofuran-C), 83.1 (tetrahydrofuran-C), 73.2 (tetrahydrofuran-C), 72.1 (tetrahydrofuran-C), $52.2 \quad\left(\mathrm{CH}_{2}-\mathrm{C}\right), \quad 50.6 \quad\left(\mathrm{CH}_{2}-\mathrm{C}\right), 42.9 \quad$ (benzyl- $\left.\mathrm{CH}_{2}-\mathrm{C}\right), 38.0 \quad\left(\mathrm{CH}_{2}-\mathrm{C}\right), 27.5 \quad\left(\mathrm{CH}_{2}-\mathrm{C}\right), 16.8$ (isopropyl- $\mathrm{CH}_{3}-\mathrm{C}$ ), 16.2 (isopropyl- $\mathrm{CH}_{3}-\mathrm{C}$ ); LRMS (ESI): $\mathrm{m} / \mathrm{z}=699[\mathrm{M}+\mathrm{Na}]^{+}$; HRMS-ESI $(\mathrm{m} / \mathrm{z})$ : Calcd. For $\mathrm{C}_{31} \mathrm{H}_{37} \mathrm{~N}_{8} \mathrm{O}_{4} \mathrm{~F}_{3} \mathrm{Cl}(\mathrm{M}+\mathrm{H})^{+}$: 677.2573; Found: 677.2585.

4.2.13.14. 1-(3-((( $\quad$ - $2 \mathrm{R}, 3 \mathrm{~S}, 4 \mathrm{R}, 5 \mathrm{R})-5-(6-((3-$ chlorobenzyl)amino)-9H-purin-9-yl)-3,4-dihydroxy tetrahydrofuran-2-yl)methyl)(isopropyl)amino)propyl)-3-(3-(trifluoromethyl)phenyl)urea $\mathbf{1 b f}$

According to the general procedure, employing $\mathbf{8 b}$ and 1-isocyanato-4-methoxylbenzene afforded compound $\mathbf{1 b f}$ as a foam solid, $74 \%$ yield, HPLC purity: $98.2 \%$, method $\mathrm{B} ;[\alpha]^{20}{ }_{\mathrm{D}}$ $=10.00(\mathrm{c} 0.53, \mathrm{MeOH}) ;{ }^{1} \mathrm{H}$ NMR $(500 \mathrm{MHz}, \mathrm{MeOD}) \delta 8.27(\mathrm{~s}, 1 \mathrm{H}$, purin-H), $8.25(\mathrm{~s}, 1 \mathrm{H}$, purin-H), $7.48(\mathrm{~d}, \mathrm{~J}=2.9 \mathrm{~Hz}, 1 \mathrm{H}, \mathrm{Ar}-\mathrm{H}), 7.42(\mathrm{~s}, 1 \mathrm{H}, \mathrm{Cl}-$ phenyl-H), 7.38 (t, J = $7.8 \mathrm{~Hz}, 1 \mathrm{H}$, 
Ar-H), 7.33-7.30 (m, 2H, Ar-H), 7.28 (brs, 1H, Ar-H), 7.21 (d, J = 7.3 Hz, 1H, Ar-H), 6.02 (d, J = $4.0 \mathrm{~Hz}, \quad 1 \mathrm{H}$, tetrahydrofuran- $\mathrm{H}), 4.88$ (brs, $2 \mathrm{H}$, benzyl- $\left.\mathrm{CH}_{2}\right), \quad 4.79-4.76 \quad(\mathrm{~m}, \quad 1 \mathrm{H}$, tetrahydrofuran- $\mathrm{H}), 4.35-4.32(\mathrm{~m}, 1 \mathrm{H}$, tetrahydrofuran- $\mathrm{H}), 4.22-4.18(\mathrm{~m}, 1 \mathrm{H}$, tetrahydrofuran- $\mathrm{H})$, 3.30-3.20 (m, 2H, $\left.\mathrm{CH}_{2}\right), 3.13$ (brs, $1 \mathrm{H}$, isopropyl-CH), $2.96\left(\mathrm{~d}, \mathrm{~J}=12.9 \mathrm{~Hz}, 1 \mathrm{H}, \mathrm{CH}_{2}-1 \mathrm{H}\right), 2.84$ (brs, $1 \mathrm{H}, \mathrm{CH}_{2}-1 \mathrm{H}$ ), 2.66 (brs, $2 \mathrm{H}, \mathrm{CH}_{2}$ ), 1.74-1.70 (m, 2H, $\left.\mathrm{CH}_{2}\right), 1.10(\mathrm{~d}, \mathrm{~J}=5.7 \mathrm{~Hz}, 3 \mathrm{H}$, isopropyl- $\left.\mathrm{CH}_{3}\right), 1.05\left(\mathrm{~d}, \mathrm{~J}=5.7 \mathrm{~Hz}, 3 \mathrm{H}\right.$, isopropyl- $\left.\mathrm{CH}_{3}\right) ;{ }^{13} \mathrm{C}$ NMR $(400 \mathrm{MHz}, \mathrm{MeOD}) 156.3$ (urea-C), 154.5 (purin-C), 152.4 (purin-C), 141.4 (purin-C), $140.5\left(\mathrm{CF}_{3}\right.$-phenyl-C), 139.6

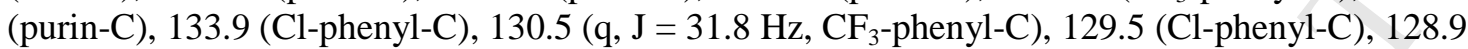
(Cl-phenyl-C), 126.9 (Cl-phenyl-C), 126.7 (Cl-phenyl-C), $125.3\left(\mathrm{CF}_{3}\right.$-phenyl-C), 124.2 (q, J = $274.3 \mathrm{~Hz}, \mathrm{CF}_{3}-\mathrm{C}$ ), 121.3 ( $\mathrm{CF}_{3}$-phenyl-C), 119.6 (purin-C), 117.3 (q, J = 3.5 Hz, $\mathrm{CF}_{3}$-phenyl-C), 114.5 (q, J = $3.9 \mathrm{~Hz}, \mathrm{CF}_{3}$-phenyl-C), 89.0 (tetrahydrofuran-C), 83.0 (tetrahydrofuran-C), 73.2 (tetrahydrofuran-C), 72.1 (tetrahydrofuran-C), $52.2\left(\mathrm{CH}_{2}-\mathrm{C}\right), 50.7\left(\mathrm{CH}_{2}-\mathrm{C}\right), 42.9$ (benzyl- $\mathrm{CH}_{2}-\mathrm{C}$ ), $37.9\left(\mathrm{CH}_{2}-\mathrm{C}\right), 27.5\left(\mathrm{CH}_{2}-\mathrm{C}\right), 16.8$ (isopropyl- $\left.\mathrm{CH}_{3}-\mathrm{C}\right), 16.1$ (isopropyl- $\left.\mathrm{CH}_{3}-\mathrm{C}\right) ; \mathrm{LRMS}$ (ESI): $\mathrm{m} / \mathrm{z}$ $=677[\mathrm{M}+\mathrm{H}]^{+}$; HRMS-ESI $(\mathrm{m} / \mathrm{z})$ : Calcd. For $\mathrm{C}_{31} \mathrm{H}_{37} \mathrm{~N}_{8} \mathrm{O}_{4} \mathrm{~F}_{3} \mathrm{Cl}(\mathrm{M}+\mathrm{H})^{+}$: 677.2573; Found: 677.2581.

\subsubsection{5.}

1-(tert-butyl)-3-(3-((((2R,3S,4R,5R)-5-(6-((3-chlorobenzyl)amino)-9H-purin-9-yl)-3,4-dihydroxy tetrahydrofuran-2-yl)methyl)(isopropyl)amino)propyl)urea $\mathbf{1 b g}$

According to the general procedure, employing $\mathbf{8 b}$ and 1-isocyanato-4-methoxylbenzene afforded compound $\mathbf{1 b g}$ as a foam solid, $76 \%$ yield, HPLC purity: $94.7 \%$, method $\mathrm{A} ;[\alpha]^{20}{ }_{\mathrm{D}}=$ 7.04 (c 0.54, MeOH); ${ }^{1} \mathrm{H}$ NMR (500 MHz, MeOD) $\delta 8.31$ (s, 1H, purin-H), $8.28(\mathrm{~s}, 1 \mathrm{H}$, purin-H), $7.43(\mathrm{~s}, 1 \mathrm{H}, \mathrm{Ar}-\mathrm{H}), 7.35-7.30(\mathrm{~m}, 2 \mathrm{H}, \mathrm{Ar}-\mathrm{H}), 7.28-7.25(\mathrm{~m}, 1 \mathrm{H}, \mathrm{Ar}-\mathrm{H}), 6.04(\mathrm{~d}, \mathrm{~J}=4.0 \mathrm{~Hz}, 1 \mathrm{H}$, tetrahydrofuran- $\mathrm{H}), 4.88$ (brs, $2 \mathrm{H}$, benzyl- $\left.\mathrm{CH}_{2}\right), 4.81-4.80(\mathrm{~m}, 1 \mathrm{H}$, tetrahydrofuran- $\mathrm{H}), 4.38-4.35$ $(\mathrm{m}, 1 \mathrm{H}$, tetrahydrofuran- $\mathrm{H}), 4.22-4.18(\mathrm{~m}, 1 \mathrm{H}$, tetrahydrofuran- $\mathrm{H}), 3.30-3.20(\mathrm{~m}, 1 \mathrm{H}$, isopropyl-CH), $3.13\left(\mathrm{t}, \mathrm{J}=6.1 \mathrm{~Hz}, 2 \mathrm{H}, \mathrm{CH}_{2}\right), 3.10-2.98\left(\mathrm{~m}, 2 \mathrm{H}, \mathrm{CH}_{2}\right), 2.72$ (brs, $\left.2 \mathrm{H}, \mathrm{CH}_{2}\right), 1.69$ (brs, $\left.2 \mathrm{H}, \mathrm{CH}_{2}\right), 1.29(\mathrm{~s}, 9 \mathrm{H}, t-\mathrm{Bu}), 1.14\left(\mathrm{~d}, \mathrm{~J}=5.7 \mathrm{~Hz}, 3 \mathrm{H}\right.$, isopropyl- $\left.\mathrm{CH}_{3}\right), 1.08(\mathrm{~d}, \mathrm{~J}=5.7 \mathrm{~Hz}$, $3 \mathrm{H}$, iso propyl- $\left.\mathrm{CH}_{3}\right) ;{ }^{13} \mathrm{C}$ NMR (400 MHz, MeOD) 159.1 (urea-C), 154.5 (purin-C), 152.4 (purin-C), 141.5 (purin-C), 139.1 (purin-C), 133.9 (Ar-C), 129.5 (Ar-C), 127.0 (Ar-C), 126.7 (Ar-C), 125.3 (Ar-C), 119.7 (purin-C), 89.2 (tetrahydrofuran-C), 82.5 (tetrahydrofuran-C), 73.1 (tetrahydrofuran-C), 72.0 (tetrahydrofuran-C), $52.0\left(\mathrm{CH}_{2}-\mathrm{C}\right), 49.3\left(\mathrm{CH}_{2}-\mathrm{C}\right), 42.9$ (benzyl- $\mathrm{CH}_{2}-\mathrm{C}$ ), $37.2\left(\mathrm{CH}_{2}-\mathrm{C}\right), 28.3\left(t-\mathrm{Bu}_{-} \mathrm{CH}_{3}-\mathrm{C}\right), 27.6\left(\mathrm{CH}_{2}-\mathrm{C}\right), 16.7$ (isopropyl- $\left.\mathrm{CH}_{3}-\mathrm{C}\right), 15.8$ (isopropyl- $\mathrm{CH}_{3}-\mathrm{C}$ ); LRMS (ESI): $\mathrm{m} / \mathrm{z}=589[\mathrm{M}+\mathrm{H}]^{+}$; HRMS-ESI $(\mathrm{m} / \mathrm{z})$ : Calcd. For $\mathrm{C}_{28} \mathrm{H}_{42} \mathrm{~N}_{8} \mathrm{O}_{4} \mathrm{Cl}(\mathrm{M}+\mathrm{H})^{+}$: 589.3012; Found: 589.3039.

\subsubsection{6.}

1-(3-((((2R,3S,4R,5R)-5-(6-((3-chlorobenzyl)amino)-9H-purin-9-yl)-3,4-dihydroxytetrahydrofura n-2-yl)methyl)(isopropyl)amino)propyl)-3-cyclohexylurea $\mathbf{1 b h}$

According to the general procedure, employing 8b and 1-isocyanato-4-methoxylbenzene afforded compound $\mathbf{1 b h}$ as a solid, $68 \%$ yield, HPLC purity: $97.3 \%$, method A; mp: $145-147^{\circ} \mathrm{C}$; $[\alpha]^{20}{ }_{\mathrm{D}}=4.71(\mathrm{c} 0.51, \mathrm{MeOH}) ;{ }^{1} \mathrm{H}$ NMR $(500 \mathrm{MHz}, \mathrm{MeOD}) \delta 8.31(\mathrm{~s}, 1 \mathrm{H}$, purin- $\mathrm{H}), 8.28(\mathrm{~s}, 1 \mathrm{H}$, purin-H), $7.43(\mathrm{~s}, 1 \mathrm{H}, \mathrm{Ar}-\mathrm{H}), 7.35-7.30(\mathrm{~m}, 2 \mathrm{H}, \mathrm{Ar}-\mathrm{H}), 7.28-7.25(\mathrm{~m}, 1 \mathrm{H}, \operatorname{Ar}-\mathrm{H}), 6.04(\mathrm{~d}, \mathrm{~J}=4.0$ $\mathrm{Hz}, 1 \mathrm{H}$, tetrahydrofuran- $\mathrm{H}), 4.88$ (brs, $2 \mathrm{H}$, benzyl- $\left.\mathrm{CH}_{2}\right), 4.81-4.80(\mathrm{~m}, 1 \mathrm{H}$, tetrahydrofuran $-\mathrm{H})$, 4.38-4.35 $(\mathrm{m}, 1 \mathrm{H}$, tetrahydrofuran- $\mathrm{H}), 4.22-4.18(\mathrm{~m}, 1 \mathrm{H}$, tetrahydrofuran- $\mathrm{H}), 3.46-3.42(\mathrm{~m}, 1 \mathrm{H}$, cyclohexyl-CH), 3.16-3.13 (m, 3H, $\mathrm{CH}_{2}$ and isopropyl- $\mathrm{CH}$ ), 3.10-2.98 (m, $\left.2 \mathrm{H}, \mathrm{CH}_{2}\right), 2.69$ (brs, $\left.1 \mathrm{H}, \mathrm{CH}_{2}\right), 1.86$ (brs, $\left.2 \mathrm{H}, \mathrm{CH}_{2}\right), 1.70-1.60\left(\mathrm{~m}, 6 \mathrm{H}, 3 \times\right.$ cyclohexyl- $\left.\mathrm{CH}_{2}\right), 1.21-1.07(\mathrm{~m}, 10 \mathrm{H}, 2 \times$ cyclohexyl- $\mathrm{CH}_{2}$ and $2 \times$ isopropyl- $\mathrm{CH}_{3}$ ); ${ }^{13} \mathrm{C}$ NMR (400 MHz, MeOD) 159.1 (urea-C), 154.5 (purin-C), 152.4 (purin-C), 148.6 (Ar-C), 141.5 (purin-C), 139.8 (purin-C), 133.9 (Ar-C), 129.5 (Ar-C), 126.9 (Ar-C), 126.7 (Ar-C), 125.3 (Ar-C), 119.7 (purin-C), 89.1 (tetrahydrofuran-C), 
82.8 (tetrahydrofuran-C), 73.1 (tetrahydrofuran-C), 72.0 (tetrahydrofuran-C), $52.0\left(\mathrm{CH}_{2}-\mathrm{C}\right), 49.8$ $\left(\mathrm{CH}_{2}-\mathrm{C}\right), 43.0$ (benzyl- $\left.\mathrm{CH}_{2}-\mathrm{C}\right), 37.5\left(\mathrm{CH}_{2}-\mathrm{C}\right), 33.2$ (cyclohexyl- $\left.\mathrm{CH}_{2}\right), 25.2\left(\right.$ cyclohexyl- $\left.\mathrm{CH}_{2}\right)$, 24.6 (cyclohexyl- $\mathrm{CH}_{2}$ ), 16.7 (isopropyl- $\mathrm{CH}_{3}-\mathrm{C}$ ), 15.9 (isopropyl- $\mathrm{CH}_{3}-\mathrm{C}$ ); LRMS (ESI): $\mathrm{m} / \mathrm{z}=$ $615[\mathrm{M}+\mathrm{H}]^{+}$; HRMS-ESI (m/z): Calcd. For $\mathrm{C}_{30} \mathrm{H}_{44} \mathrm{~N}_{8} \mathrm{O}_{4} \mathrm{Cl}(\mathrm{M}+\mathrm{H})^{+}$: 615.3169; Found: 615.3186.

4.2.14. General procedure for the preparation of compounds 9-12

To a stirred solution of compound 5-8 in DCM $(6 \mathrm{~mL})$ was added TFA $(1 \mathrm{~mL})$ and $\mathrm{H}_{2} \mathrm{O}(0.5$ $\mathrm{mL}$ ) at $0{ }^{\circ} \mathrm{C}$. The mixture was stirred overnight and concentrated. The residue was purified by preparing TLC (DCM : $\mathrm{MeOH}: \mathrm{NH}_{3} \mathrm{H}_{2} \mathrm{O}=70: 10: 1$ ) to yield compound 9-12.

\subsubsection{1.}

(2R,3R,4S,5R)-2-(6-((3-fluorobenzyl)amino)-9H-purin-9-yl)-5-((isopropylamino)methyl) tetrahydrofuran-3,4-diol 9a

According to the general procedure, employing 5a afforded compound $9 \mathbf{a}$ as a foam solid, $90 \%$ yield, HPLC purity: $97.8 \%$, method $\mathrm{C} ;[\alpha]^{20}{ }_{\mathrm{D}}=-2.55(\mathrm{c} 0.67, \mathrm{MeOH}) ;{ }^{1} \mathrm{H}$ NMR $(500 \mathrm{MHz}$, MeOD) $\delta 8.30(\mathrm{~s}, 1 \mathrm{H}$, purin-H), $8.26(\mathrm{~s}, 1 \mathrm{H}$, purin-H), 7.36-7.33 (m, 1H, Ar-H), $7.22(\mathrm{~d}, \mathrm{~J}=7.5$ $\mathrm{Hz}, 1 \mathrm{H}, \mathrm{Ar}-\mathrm{H}), 7.15(\mathrm{~d}, \mathrm{~J}=9.8 \mathrm{~Hz}, 1 \mathrm{H}, \mathrm{Ar}-\mathrm{H}), 7.00-6.98(\mathrm{~m}, 1 \mathrm{H}, \mathrm{Ar}-\mathrm{H}), 6.01(\mathrm{~d}, \mathrm{~J}=3.8 \mathrm{~Hz}, 1 \mathrm{H}$, tetrahydrofuran- $\mathrm{H}), \quad 4.85-4.83\left(\mathrm{~m}, 2 \mathrm{H}\right.$, benzyl- $\left.\mathrm{CH}_{2}\right), 4.37(\mathrm{dd}, \mathrm{J}=4.2,4.7 \mathrm{~Hz}, 1 \mathrm{H}$, tetrahydrofuran- $\mathrm{H}), 4.28-4.26(\mathrm{~m}, 1 \mathrm{H}$, tetrahydrofuran $-\mathrm{H}), 3.24-3.20(\mathrm{~m}, 1 \mathrm{H}$, isopropyl- $\mathrm{CH})$, 3.15-3.08 (m, 2H, $\left.\mathrm{CH}_{2}\right), 1.20-1.18\left(\mathrm{~m}, 6 \mathrm{H}\right.$, isopropyl- $\left.\mathrm{CH}_{3}\right) ;{ }^{13} \mathrm{C}$ NMR $(500 \mathrm{MHz}, \mathrm{MeOD}) \delta 162.8$ (d, J = $245 \mathrm{~Hz}$, Ar-C), 154.7 (purin-C), 152.5 (puirn-C), 142.0 (puirn-C), 140.4 (puirn-C), 129.8 (Ar-C), 128.7 (Ar-C), 122.7 (d, J = $3 \mathrm{~Hz}$, Ar-C), 120.0 (purin-C), 113.6 (d, J = 21.6 Hz, Ar-C), $113.3(\mathrm{~d}, \mathrm{~J}=21.6 \mathrm{~Hz}$, Ar-C), 89.7 (tetrahydrofuran-C), 82.4 (tetrahydrofuran-C), 73.1 (tetrahydrofuran-C), 72.0 (tetrahydrofuran-C), $49.3\left(\mathrm{CH}_{2}-\mathrm{C}\right), 48.0$ (isopropyl-CH-C), 19.9 (isopropyl- $\mathrm{CH}_{3}-\mathrm{C}$ ), 19.8 (isopropyl- $\mathrm{CH}_{3}-\mathrm{C}$ ); LRMS (ESI): $\mathrm{m} / \mathrm{z}=439[\mathrm{M}+\mathrm{Na}]^{+}$; HRMS-ESI $(\mathrm{m} / \mathrm{z})$ : Calcd. For $\mathrm{C}_{20} \mathrm{H}_{26} \mathrm{~N}_{6} \mathrm{O}_{3} \mathrm{~F}(\mathrm{M}+\mathrm{H})^{+}$: 417.2045; Found: 417.2062.

4.2.14.2. (2R,3R,4S,5R)-2-(6-((3-chlorobenzyl)amino)-9H-purin-9-yl)-5-((isopropylamino) methyl) tetrahydrofuran-3,4-diol 9b

According to the general procedure, employing $\mathbf{5 b}$ afforded compound $\mathbf{9 b}$ as an oil, $94 \%$ yield, HPLC purity: 97.9\%, method $\mathrm{C} ;[\alpha]^{20}{ }_{\mathrm{D}}=-4.13$ (c $\left.0.99, \mathrm{MeOH}\right) ;{ }^{1} \mathrm{H}$ NMR $(500 \mathrm{MHz}$, MeOD) $\delta 8.30(\mathrm{~s}, 1 \mathrm{H}$, purin-H), $8.26(\mathrm{~s}, 1 \mathrm{H}$, purin-H), $7.42(\mathrm{~s}, 1 \mathrm{H}, \mathrm{Ar}-\mathrm{H}), 7.33-7.26(\mathrm{~m}, 3 \mathrm{H}$, Ar-H), $6.01\left(\mathrm{~d}, \mathrm{~J}=3.8 \mathrm{~Hz}, 1 \mathrm{H}\right.$, tetrahydrofuran-H), 4.85-4.83 (m, 2H, benzyl- $\left.\mathrm{CH}_{2}\right), 4.35(\mathrm{dd}, \mathrm{J}=$ 4.2, $4.7 \mathrm{~Hz}, 1 \mathrm{H}$, tetrahydrofuran-H), 4.18-4.16 (m, $1 \mathrm{H}$, tetrahydrofuran- $\mathrm{H}), 3.20-3.04(\mathrm{~m}, 3 \mathrm{H}$, $\mathrm{CH}_{2}$ and isopropyl-CH), $1.19-1.17\left(\mathrm{~m}, 6 \mathrm{H}\right.$, isopropyl- $\left.\mathrm{CH}_{3}\right) ;{ }^{13} \mathrm{C}$ NMR $(500 \mathrm{MHz}, \mathrm{MeOD}) \delta 154.6$ (purin-C), 152.5 (purin-C), 141.5 (purin-C), 140.4 (purin-C), 133.9 (Ar-C), 129.6 (Ar-C), 129.4 (Ar-C), 126.9 (Ar-C), 126.7 (Ar-C), 125.4 (Ar-C), 120.0 (purin-C), 89.7 (tetrahydrofuran-C), 82.6 (tetrahydrofuran-C), 73.1 (tetrahydrofuran-C), 72.0 (tetrahydrofuran-C), $49.2\left(\mathrm{CH}_{2}-\mathrm{C}\right), 48.2$ (isopropyl-CH-C), 20.1 (isopropyl- $\mathrm{CH}_{3}-\mathrm{C}$ ); LRMS (ESI): $\mathrm{m} / \mathrm{z}=433[\mathrm{M}+\mathrm{H}]^{+} ;$HRMS-ESI (m/z): Calcd. For $\mathrm{C}_{20} \mathrm{H}_{26} \mathrm{~N}_{6} \mathrm{O}_{3} \mathrm{Cl}(\mathrm{M}+\mathrm{H})^{+}$: 433.1749; Found: 433.1760 .

4.2.14.3. methyl 3-((((2R,3S,4R,5R)-5-(6-((3-fluorobenzyl)amino)-9H-purin-9-yl)-3,4-dihydroxytetrahydrofuran-2 -yl)methyl)(isopropyl)amino)propanoate 10a

According to the general procedure, employing 6a afforded compound 10a as a foam solid, 93\% yield, $[\alpha]_{\mathrm{D}}^{20}=-7.56(\mathrm{c} 1.73, \mathrm{MeOH}) ;{ }^{1} \mathrm{H}$ NMR (500 MHz, MeOD) $\delta 8.30(\mathrm{~s}, 1 \mathrm{H}$, purin-H), $8.26(\mathrm{~s}$, 1H, purin-H), 7.36-7.33 (m, 1H, Ar-H), 7.22 (d, J = 7.5 Hz, 1H, Ar-H), 7.15 (d, J = 9.8 Hz, 1H, Ar-H), 7.00-6.98 (m, 1H, Ar-H), 6.01 (d, J = 3.8 Hz, 1H, tetrahydrofuran-H), 4.85-4.83 (m, 2H, 
benzyl- $\left.\mathrm{CH}_{2}\right), 4.35(\mathrm{dd}, \mathrm{J}=4.2,4.7 \mathrm{~Hz}, 1 \mathrm{H}$, tetrahydrofuran- $\mathrm{H}), 4.13-4.10(\mathrm{~m}, 1 \mathrm{H}$, tetrahydrofuran- $\mathrm{H}), 3.63\left(\mathrm{~s}, 3 \mathrm{H}, \mathrm{CH}_{3} \mathrm{O}\right), 3.02-2.92\left(\mathrm{~m}, 2 \mathrm{H}\right.$, isopropyl- $\mathrm{CH}$ and $\left.\mathrm{CH}_{2}-1 \mathrm{H}\right), 2.84-2.75$ $\left(\mathrm{m}, 3 \mathrm{H}, \mathrm{CH}_{2}\right.$ and $\left.\mathrm{CH}_{2}-1 \mathrm{H}\right), 2.48\left(\mathrm{t}, \mathrm{J}=7.0 \mathrm{~Hz}, 2 \mathrm{H}, \mathrm{CH}_{2}\right), 1.06\left(\mathrm{~d}, \mathrm{~J}=6.0 \mathrm{~Hz}, 3 \mathrm{H}\right.$, isopropyl- $\left.\mathrm{CH}_{3}\right)$, $1.01\left(\mathrm{~d}, \mathrm{~J}=6.0 \mathrm{~Hz}, 3 \mathrm{H}\right.$, isopropyl- $\left.\mathrm{CH}_{3}\right) ;{ }^{13} \mathrm{C} \mathrm{NMR}(500 \mathrm{MHz}, \mathrm{MeOD}) \delta 173.5$ (ester-C), $162.5(\mathrm{~d}$, $\mathrm{J}=245.4 \mathrm{~Hz}$, Ar-C), 154.6 (purin-C), 152.5 (purin-C), 142.0 (purin-C), 139.9 (purin-C), 129.9 (Ar-C), 129.8 (Ar-C), 122.7 (d, J = $3 \mathrm{~Hz}$, Ar-C), 119.6 (purin-C), 113.7 (d, J = 21.6 Hz, Ar-C), $113.3(\mathrm{~d}, \mathrm{~J}=21.6 \mathrm{~Hz}, \quad$ Ar-C), 88.9 (tetrahydrofuran-C), 83.8 (tetrahydrofuran-C), 73.1 (tetrahydrofuran-C), 71.7 (tetrahydrofuran-C), $51.9(\mathrm{MeO}-\mathrm{C}), 51.1\left(\mathrm{CH}_{2}-\mathrm{C}\right), 50.6\left(\mathrm{CH}_{2}-\mathrm{C}\right), 49.1$ (isopropyl-CH-C), 33.4 (isopropyl- $\mathrm{CH}_{3}-\mathrm{C}$ ), 17.6 (isopropyl- $\mathrm{CH}_{3}-\mathrm{C}$ ), 16.3; LRMS (ESI): $\mathrm{m} / \mathrm{z}=$ $525[\mathrm{M}+\mathrm{Na}]^{+}$; HRMS-ESI $(\mathrm{m} / \mathrm{z})$ : Calcd. For $\mathrm{C}_{24} \mathrm{H}_{32} \mathrm{~N}_{6} \mathrm{O}_{5} \mathrm{~F}(\mathrm{M}+\mathrm{H})^{+}$: 503.2413; Found: 503.2429 .

4.2.14.4.

methyl

3-((( (2R,3S,4R,5R)-5-(6-((3-chlorobenzyl)amino)-9H-purin-9-yl)-3,4-dihydroxytetrahydrofuran2-yl)methyl)(isopropyl)amino)propanoate $\mathbf{1 0 b}$

According to the general procedure, employing $\mathbf{6 b}$ afforded compound $\mathbf{1 0 b}$ as a foam solid, $85 \%$ yield, $[\alpha]^{20}{ }_{\mathrm{D}}=-2.65(\mathrm{c} 0.57, \mathrm{MeOH}) ;{ }^{1} \mathrm{H}$ NMR $(500 \mathrm{MHz}, \mathrm{MeOD}) \delta 8.30(\mathrm{~s}, 2 \mathrm{H}$, purin-C), $7.42(\mathrm{~s}, 1 \mathrm{H}, \mathrm{Ar}-\mathrm{H}), 7.33-7.26(\mathrm{~m}, 3 \mathrm{H}, \mathrm{Ar}-\mathrm{H}), 6.01(\mathrm{~d}, \mathrm{~J}=3.8 \mathrm{~Hz}, 1 \mathrm{H}$, tetrahydrofuran $-\mathrm{H})$, 4.85-4.83 (m, 2H, benzyl- $\left.\mathrm{CH}_{2}\right), 4.35(\mathrm{dd}, \mathrm{J}=4.2,4.7 \mathrm{~Hz}, 1 \mathrm{H}$, tetrahydrofuran- $\mathrm{H}), 4.13-4.10(\mathrm{~m}$, $1 \mathrm{H}$, tetrahydrofuran- $\mathrm{H}), 3.63\left(\mathrm{~s}, 3 \mathrm{H}, \mathrm{CH}_{3} \mathrm{O}\right), 3.02-2.94\left(\mathrm{~m}, 2 \mathrm{H}\right.$, ispropyl- $\mathrm{CH}$ and $\left.\mathrm{CH}_{2}-1 \mathrm{H}\right)$, 2.85-2.82 (m, 3H, $\mathrm{CH}_{2}$ and $\left.\mathrm{CH}_{2}-1 \mathrm{H}\right), 2.50-2.47\left(\mathrm{t}, \mathrm{J}=7.0 \mathrm{~Hz}, 2 \mathrm{H}, \mathrm{CH}_{2}\right), 1.06(\mathrm{~d}, \mathrm{~J}=6.6 \mathrm{~Hz}, 3 \mathrm{H}$, isopropyl- $\left.\mathrm{CH}_{3}\right), 1.01\left(\mathrm{~d}, \mathrm{~J}=6.6 \mathrm{~Hz}, 3 \mathrm{H}\right.$, isopropyl- $\left.\mathrm{CH}_{3}\right) ;{ }^{13} \mathrm{C} \mathrm{NMR}(500 \mathrm{MHz}, \mathrm{MeOD}) \delta 173.5$ (ester-C), 154.6 (purin-C), 152.5 (purin-C), 141.5 (purin-C), 140.0 (purin-C), 133.9 (Ar-C), 129.6 (Ar-C), 129.4 (Ar-C), 127.0 (Ar-C), 126.7 (Ar-C), 125.4 (Ar-C), 119.8 (purin-C), 88.9 (tetrahydrofuran-C), 83.8 (tetrahydrofuran-C), 73.1 (tetrahydrofuran-C), 71.7 (tetrahydrofuran-C), 51.9 (MeO-C), $51.2\left(\mathrm{CH}_{2}-\mathrm{C}\right), 50.6\left(\mathrm{CH}_{2}-\mathrm{C}\right), 46.1$ (isopropyl-CH-C), $33.3\left(\mathrm{CH}_{2}-\mathrm{C}\right), 17.5$ (isopropyl- $\mathrm{CH}_{3}-\mathrm{C}$ ), 16.3 (isopropyl- $\mathrm{CH}_{3}-\mathrm{C}$ ); $\mathrm{LRMS}(\mathrm{ESI})$ : $\mathrm{m} / \mathrm{z}=519[\mathrm{M}+\mathrm{H}]^{+}$.

4.2.14.5. (2R,3R,4S,5R)-2-(6-((3-fluorobenzyl)amino)-9H-purin-9-yl)-5-(((3-hydroxypropyl) (isopropyl)amino)methyl)tetrahydrofuran-3,4-diol 11a

According to the general procedure, employing $\mathbf{7 a}$ afforded compound 11a as a white solid, $88 \%$ yield, HPLC purity: $99.5 \%$, method A; mp: $152-154{ }^{\circ} \mathrm{C} ;[\alpha]^{20}=0.45(\mathrm{c} 0.67, \mathrm{MeOH}) ;{ }^{1} \mathrm{H}$ NMR $(500 \mathrm{MHz}, \mathrm{MeOD}) \delta 8.30(\mathrm{~s}, 2 \mathrm{H}$, purin-H), $8.26(\mathrm{~s}, 1 \mathrm{H}$, purin-H), 7.36-7.33 (m, 1H, Ar-H), 7.22 $(\mathrm{d}, \mathrm{J}=7.5 \mathrm{~Hz}, 1 \mathrm{H}, \mathrm{Ar}-\mathrm{H}), 7.15(\mathrm{~d}, \mathrm{~J}=9.8 \mathrm{~Hz}, 1 \mathrm{H}, \mathrm{Ar}-\mathrm{H}), 7.00-6.98(\mathrm{~m}, 1 \mathrm{H}, \mathrm{Ar}-\mathrm{H}), 6.01(\mathrm{~d}, \mathrm{~J}=$ $3.8 \mathrm{~Hz}, 1 \mathrm{H}$, tetrahydrofuran- $\mathrm{H}), 4.85-4.83\left(\mathrm{~m}, 2 \mathrm{H}\right.$, benzyl- $\left.\mathrm{CH}_{2}\right), 4.35(\mathrm{dd}, \mathrm{J}=4.2,4.7 \mathrm{~Hz}, 1 \mathrm{H}$, tetrahydrofuran- $\mathrm{H}), 4.19-4.18(\mathrm{~m}, 1 \mathrm{H}$, tetrahydrofuran- $\mathrm{H}), 3.65\left(\mathrm{t}, \mathrm{J}=5.5 \mathrm{~Hz}, 2 \mathrm{H}, \mathrm{CH}_{2}\right)$, 3.17-3.13 (m, $1 \mathrm{H}$, isopropyl-CH), 2.99-2.96 (m, $\left.1 \mathrm{H}, \mathrm{CH}_{2}-1 \mathrm{H}\right), 2.89-2.85\left(\mathrm{~m}, 1 \mathrm{H}, \mathrm{CH}_{2}-1 \mathrm{H}\right), 2.73$ (brs, $\left.2 \mathrm{H}, \mathrm{CH}_{2}\right) 1.72\left(\mathrm{t}, \mathrm{J}=6.8 \mathrm{~Hz}, 2 \mathrm{H}, \mathrm{CH}_{2}\right), 1.10\left(\mathrm{~d}, \mathrm{~J}=6.5 \mathrm{~Hz}, 3 \mathrm{H}\right.$, isopropyl- $\left.\mathrm{CH}_{3}\right), 1.04(\mathrm{~d}, \mathrm{~J}=$ $6.5 \mathrm{~Hz}, 3 \mathrm{H}$, isopropyl- $\left.\mathrm{CH}_{3}\right) ;{ }^{13} \mathrm{C}$ NMR $(500 \mathrm{MHz}, \mathrm{MeOD}) \delta 162.5(\mathrm{~d}, \mathrm{~J}=245 \mathrm{~Hz}, \mathrm{Ar}-\mathrm{C}), 154.6$ (purin-C), 152.5 (purin-C), 142.0 (purin-C), 139.9 (purin-C), 129.8 (Ar-C), 129.7 (Ar-C), 122.7 (Ar-C), 119.7 (purin-C), $113.6(\mathrm{~d}, \mathrm{~J}=21.6 \mathrm{~Hz}$, Ar-C), 113.5 (d, $\mathrm{J}=21.6 \mathrm{~Hz}$, Ar-C), 89.1 (tetrahydrofuran-C), 83.0 (tetrahydrofuran-C), 73.2 (tetrahydrofuran-C), 72.1 (tetrahydrofuran-C), $60.8\left(\mathrm{CH}_{2}-\mathrm{C}\right), \quad 52.2\left(\mathrm{CH}_{2}-\mathrm{C}\right), \quad 50.9\left(\mathrm{CH}_{2}-\mathrm{C}\right), 48.9$ (isopropyl-CH-C), $29.5 \quad\left(\mathrm{CH}_{2}-\mathrm{C}\right), \quad 16.8$ (isopropyl- $\mathrm{CH}_{3}-\mathrm{C}$ ), 16.1 (isopropyl- $\mathrm{CH}_{3}-\mathrm{C}$ ); LRMS (ESI): $\mathrm{m} / \mathrm{z}=497[\mathrm{M}+\mathrm{Na}]^{+}$; HRMS-ESI $(\mathrm{m} / \mathrm{z})$ : Calcd. For $\mathrm{C}_{23} \mathrm{H}_{32} \mathrm{~N}_{6} \mathrm{O}_{4} \mathrm{~F}(\mathrm{M}+\mathrm{H})^{+}$: 475.2464; Found: 475.2458 .

4.2.14.6. (2R,3R,4S,5R)-2-(6-((3-chlorobenzyl)amino)-9H-purin-9-yl)-5-(((3-hydroxypropyl) (isopropyl)amino)methyl)tetrahydrofuran-3,4-diol 11b 
According to the general procedure, employing $\mathbf{7 b}$ afforded compound $\mathbf{1 1 b}$ as a white solid, $89 \%$ yield, HPLC purity: $92.7 \%$, method $\mathrm{A}$; mp: $144-147^{\circ} \mathrm{C} ;[\alpha]^{20}{ }_{\mathrm{D}}=-0.43(\mathrm{c} 0.70, \mathrm{MeOH}) ;{ }^{1} \mathrm{H}$ NMR (500 MHz, MeOD) $\delta 8.30(\mathrm{~s}, 1 \mathrm{H}$, purin-H), $8.26(\mathrm{~s}, 1 \mathrm{H}$, purin-H), $7.42(\mathrm{~s}, 1 \mathrm{H}$, Ar-H), 7.33-7.26 $(\mathrm{m}, 3 \mathrm{H}, \operatorname{Ar}-\mathrm{H}), 6.01(\mathrm{~d}, \mathrm{~J}=3.8 \mathrm{~Hz}, 1 \mathrm{H}$, tetrahydrofuran- $\mathrm{H}), 4.85-4.83(\mathrm{~m}, 2 \mathrm{H}$, benzyl- $\left.\mathrm{CH}_{2}\right), 4.76$ (brs, $1 \mathrm{H}$, tetrahydrofuran- $\left.\mathrm{H}\right), 4.30-4.28(\mathrm{~m}, 1 \mathrm{H}$, tetrahydrofuran- $\mathrm{H}), 4.18-4.16$ $(\mathrm{m}, 1 \mathrm{H}$, tetrahydrofuran- $\mathrm{H}), 3.62\left(\mathrm{t}, \mathrm{J}=6.8 \mathrm{~Hz}, 2 \mathrm{H}, \mathrm{CH}_{2}\right), 3.15-3.08(\mathrm{~m}, 1 \mathrm{H}$, isopropyl- $\mathrm{CH})$, 2.94-2.92 (m, $\left.1 \mathrm{H}, \mathrm{CH}_{2}-1 \mathrm{H}\right), 2.82-2.81\left(\mathrm{~m}, 1 \mathrm{H}, \mathrm{CH}_{2}-1 \mathrm{H}\right), 2.68$ (brs, $\left.2 \mathrm{H}, \mathrm{CH}_{2}\right), 1.69(\mathrm{t}, \mathrm{J}=6.9 \mathrm{~Hz}$, $\left.2 \mathrm{H}, \mathrm{CH}_{2}\right), 1.06\left(\mathrm{~d}, \mathrm{~J}=6.5 \mathrm{~Hz}, 3 \mathrm{H}\right.$, isopropyl- $\left.\mathrm{CH}_{3}\right), 1.00\left(\mathrm{~d}, \mathrm{~J}=6.5 \mathrm{~Hz}, 3 \mathrm{H}\right.$, isopropyl- $\left.\mathrm{CH}_{3}\right) ;{ }^{13} \mathrm{C}$ NMR (500 MHz, MeOD) $\delta 154.6$ (purin-C), 152.5 (purin-C), 141.5 (purin-C), 139.9 (purin-C), 133.9 (Ar-C), 129.6 (Ar-C), 129.4 (Ar-C), 127.0 (Ar-C), 126.8 (Ar-C), 125.4 (Ar-C), 119.7 (purin-C), 89.1 (tetrahydrofuran-C), 83.2 (tetrahydrofuran-C), 73.2 (tetrahydrofuran-C), 72.1 (tetrahydrofuran-C), $60.9\left(\mathrm{CH}_{2}-\mathrm{C}\right), 52.1\left(\mathrm{CH}_{2}-\mathrm{C}\right), 50.7\left(\mathrm{CH}_{2}-\mathrm{C}\right), 48.2$ (isopropyl-CH-C), 29.7 $\left(\mathrm{CH}_{2}-\mathrm{C}\right.$ ), 16.9 (isopropyl- $\mathrm{CH}_{3}-\mathrm{C}$ ), 16.1 (isopropyl- $\mathrm{CH}_{3}-\mathrm{C}$ ); LRMS (ESI): $\mathrm{m} / \mathrm{z}=513\left[\mathrm{M}+\mathrm{Na}^{+}\right.$; HRMS-ESI (m/z): Calcd. For $\mathrm{C}_{23} \mathrm{H}_{32} \mathrm{~N}_{6} \mathrm{O}_{4} \mathrm{Cl}(\mathrm{M}+\mathrm{H})^{+}:$491.2168; Found: 491.2170.

\subsubsection{7.}

(2R,3S,4R,5R)-2-(((3-aminopropyl)(isopropyl)amino)methyl)-5-(6-((3-fluorobenzyl)amino)-9Hpurin-9-yl)tetrahydrofuran-3,4-diol 12a

According to the general procedure, employing 8a afforded compound 12a as an oil, 79\% yield, $[\alpha]^{20}{ }_{\mathrm{D}}=10.53(\mathrm{c} 1.02, \mathrm{MeOH}) ;{ }^{1} \mathrm{H}$ NMR $(500 \mathrm{MHz}, \mathrm{MeOD}) \delta 8.30(\mathrm{~s}, 1 \mathrm{H}$, purin-H), 8.26 (s, 1H, purin-H), 7.36-7.33 (m, 1H, Ar-H), $7.22(\mathrm{~d}, \mathrm{~J}=7.5 \mathrm{~Hz}, 1 \mathrm{H}, \operatorname{Ar}-\mathrm{H}), 7.15(\mathrm{~d}, \mathrm{~J}=9.8 \mathrm{~Hz}, 1 \mathrm{H}$, Ar-H), 7.00-6.98 (m, 1H, Ar-H), $6.01(\mathrm{~d}, \mathrm{~J}=3.8 \mathrm{~Hz}, 1 \mathrm{H}$, tetrahydrofuran-H), 4.82 (brs, $2 \mathrm{H}$, benzyl- $\left.\mathrm{CH}_{2}\right), 4.81-4.79(\mathrm{~m}, 1 \mathrm{H}$, tetrahydrofuran- $\mathrm{H}), 4.40-4.38(\mathrm{~m}, 1 \mathrm{H}$, tetrahydrofuran- $\mathrm{H})$, 4.32-4.30 (m, $1 \mathrm{H}$, tetrahydrofuran-), 3.12-2.98 (m, $7 \mathrm{H}, 3 \times \mathrm{CH}_{2}$ and isopropyl- $\left.\mathrm{CH}\right), 1.92-1.90$ (brs, $\left.2 \mathrm{H}, \mathrm{CH}_{2}\right), 1.19\left(\mathrm{~d}, \mathrm{~J}=5.3 \mathrm{~Hz}, 3 \mathrm{H}\right.$, isopropyl- $\left.\mathrm{CH}_{3}\right), 1.13\left(\mathrm{~d}, \mathrm{~J}=5.3 \mathrm{~Hz}, 3 \mathrm{H}\right.$, isopropyl- $\left.\mathrm{CH}_{3}\right)$; ${ }^{13} \mathrm{C}$ NMR $(500 \mathrm{MHz}, \mathrm{MeOD}) \delta 162.5(\mathrm{~d}, \mathrm{~J}=245.1 \mathrm{~Hz}, \mathrm{Ar}-\mathrm{C}), 154.7$ (purin-C), 152.6 (purin-C), 142.0 (purin-C), 139.8 (purin-C), 129.8 (d, J = 7.9 Hz, Ar-C), 129.4 (Ar-C), 122.8 (d, J = 3.2 Hz, Ar-C), 119.8 (purin-C), $113.6(\mathrm{~d}, \mathrm{~J}=21.8 \mathrm{~Hz}, \mathrm{Ar}-\mathrm{C}), 113.3(\mathrm{~d}, \mathrm{~J}=21.6 \mathrm{~Hz}, \mathrm{Ar}-\mathrm{C}), 89.5$ (tetrahydrofuran-C), 81.4 (tetrahydrofuran-C), 73.1 (tetrahydrofuran-C), 72.1 (tetrahydrofuran-C), $52.1\left(\mathrm{CH}_{2}-\mathrm{C}\right), 48.8$ (isopropyl-CH-C), $29.4\left(\mathrm{CH}_{2}-\mathrm{C}\right), 23.3\left(\mathrm{CH}_{2}-\mathrm{C}\right), 16.3$ (isopropyl- $\left.\mathrm{CH}_{3}-\mathrm{C}\right), 15.4$ (isopropyl- $\mathrm{CH}_{3}-\mathrm{C}$ ); LRMS (ESI): $\mathrm{m} / \mathrm{z}=496[\mathrm{M}+\mathrm{Na}]^{+}$; HRMS-ESI $(\mathrm{m} / \mathrm{z}$ ): Calcd. For $\mathrm{C}_{23} \mathrm{H}_{33} \mathrm{~N}_{7} \mathrm{O}_{3} \mathrm{~F}(\mathrm{M}+\mathrm{H})^{+}$: 474.2623; Found: 474.2625.

\subsubsection{8.}

(2R,3S,4R,5R)-2-(((3-aminopropyl)(isopropyl)amino)methyl)-5-(6-((3-chlorobenzyl)amino)-9Hpurin-9-yl)tetrahydrofuran-3,4-diol 12b

According to the general procedure, employing $\mathbf{8 b}$ afforded compound $\mathbf{1 2 b}$ as an oil, $85 \%$ yield, $[\alpha]^{20}{ }_{\mathrm{D}}=8.74(\mathrm{c} 1.09, \mathrm{MeOH}) ;{ }^{1} \mathrm{H}$ NMR $(500 \mathrm{MHz}, \mathrm{MeOD}) \delta 8.32(\mathrm{~s}, 1 \mathrm{H}$, purin-H), $8.27(\mathrm{~s}, 1 \mathrm{H}$, purin-H), $7.42(\mathrm{~s}, \quad 1 \mathrm{H}$, Ar-H), 7.34-7.27 (m, 3H, Ar-H), $6.04(\mathrm{~d}, \mathrm{~J}=4.1 \mathrm{~Hz}, 1 \mathrm{H}$, tetrahydrofuran- $\mathrm{H}), 4.85$ (brs, $2 \mathrm{H}$, benzyl- $\left.\mathrm{CH}_{2}\right), 4.81-4.80(\mathrm{~m}, 1 \mathrm{H}$, tetrahydrofuran- $\mathrm{H}), 4.34(\mathrm{t}, \mathrm{J}=$ $5.3 \mathrm{~Hz}, 1 \mathrm{H}$, tetrahydrofuran- $\mathrm{H}), 4.25-4.20(\mathrm{~m}, 1 \mathrm{H}$, tetrahydrofuran- $\mathrm{H}), 3.20-3.15(\mathrm{~m}, 1 \mathrm{H}$, isopropyl- $\mathrm{CH}), 3.07\left(\mathrm{t}, \mathrm{J}=6.0 \mathrm{~Hz}, 2 \mathrm{H}, \mathrm{CH}_{2}\right), 2.95-2.78\left(\mathrm{~m}, 4 \mathrm{H}, 2 \times \mathrm{CH}_{2}\right), 1.81-1.78(\mathrm{~m}, 2 \mathrm{H}$, $\left.\mathrm{CH}_{2}\right), 1.10\left(\mathrm{~d}, \mathrm{~J}=6.0 \mathrm{~Hz}, 3 \mathrm{H}\right.$, isopropyl- $\left.\mathrm{CH}_{3}\right), 1.04\left(\mathrm{~d}, \mathrm{~J}=6.0 \mathrm{~Hz}, 3 \mathrm{H}\right.$, isopropyl- $\left.\mathrm{CH}_{3}\right) ;{ }^{13} \mathrm{C} \mathrm{NMR}$ (500 MHz, MeOD) $\delta 154.6$ (purin-C), 152.5 (purin-C), 141.5 (purin-C), 139.9 (purin-C), 133.9 (Ar-C), 129.6 (Ar-C), 129.4 (Ar-C), 127.0 (Ar-C), 126.8 (Ar-C), 125.4 (Ar-C), 119.7 (purin-C), 89.1 (tetrahydrofuran-C), 82.3 (tetrahydrofuran-C), 73.2 (tetrahydrofuran-C), 72.1 (tetrahydrofuran-C), $52.0\left(\mathrm{CH}_{2}-\mathrm{C}\right), 49.1$ (isopropyl-CH-C), $39.6\left(\mathrm{CH}_{2}-\mathrm{C}\right), 29.4\left(\mathrm{CH}_{2}-\mathrm{C}\right), 23.5$ $\left(\mathrm{CH}_{2}-\mathrm{C}\right.$ ), 16.6 (isopropyl- $\mathrm{CH}_{3}-\mathrm{C}$ ), 15.4 (isopropyl- $\mathrm{CH}_{3}-\mathrm{C}$ ); LRMS (ESI): $\mathrm{m} / \mathrm{z}=512\left[\mathrm{M}+\mathrm{Na}^{+}\right.$; HRMS-ESI (m/z): Calcd. For $\mathrm{C}_{23} \mathrm{H}_{33} \mathrm{~N}_{7} \mathrm{O}_{3} \mathrm{Cl}(\mathrm{M}+\mathrm{H})^{+}$: 490.2328; Found: 490.2327. 


\subsection{HPLC purity determination}

All samples were performed on an Agilent 1260 HPLC-UV system, using Method A, B, or C as follows. Solvent $\mathrm{A}=$ Acetonitrile; solvent $\mathrm{B}=$ Tetramethylammonium hydroxide solution, solvent $\mathrm{C}=$ Methonal; column (Waters Xterra RP18, $4.6 \mathrm{~mm} \times 250 \mathrm{~mm}, 5 \mu \mathrm{m}$ ), $40{ }^{\circ} \mathrm{C}$; UV at 254 nm.

Preparation of solvent B: To a stirred solution tetramethylammonium hydroxide $(4.53 \mathrm{~g})$ in $\mathrm{H}_{2} \mathrm{O}(1000 \mathrm{~mL})$ was added triethylamine $(0.1 \mathrm{~mL})$, and then adjusted to $\mathrm{pH} 9.0$ by phosphoric acid.

Method A: Solvent A : Solvent B = $50: 50$, flow: $0.7 \mathrm{~mL} / \mathrm{min}, 16 \mathrm{~min}$;

Method B: Solvent A : Solvent B = $50: 50$, flow: $1.5 \mathrm{~mL} / \mathrm{min}, 16 \mathrm{~min}$;

Method C: Solvent C : Solvent B = 60 : 40, flow: $1.2 \mathrm{~mL} / \mathrm{min}, 20 \mathrm{~min}$.

\subsection{Anti-ZIKV Assay}

The reference drug Sinefungin and EPZ004777 were purchased from STEMCELL Technologies. The ZIKV was obtained from the Institute of Microbiology, Chinese Academy of Sciences, Beijing, China.

BHK cells were seeded into 96-well plate using DMEM supplemented with 10\% FBS and penicillin and streptomycin (100 units $/ \mathrm{ml}$ and $100 \mu \mathrm{g} / \mathrm{ml}$, respectively) at a density of 5,000 cells per well. After $24 \mathrm{~h}$ incubation at $37^{\circ} \mathrm{C}$ with $5 \% \mathrm{CO}_{2}$, original medium was changed into maintenance medium (DMEM supplemented with $2 \%$ FBS and P/S) in a volume of $100 \mu \mathrm{L}$. Zika virus stocks propagated in Vero cells were diluted to ${ }_{100 \mathrm{TCID}_{50}}$ and added $50 \mu \mathrm{L}$ to each virus control and administered well. The compounds that diluted from $50 \mu \mathrm{M}$ by 3 -fold dilution in maintenance medium were added to administered wells. The wells were supplemented with maintenance medium to $200 \mu \mathrm{L}$. The plates were incubated for 8 days until the CPE reached $100 \%$. The cell viability was measured by Cell Titer-Glo® luminescent cell viability kit according to the manufacturer's instructions. The $\mathrm{IC}_{50}$ values were calculated by Origin 8.0.

\subsection{Cytotoxicity determination}

Various concentrations of compounds from $200 \mu \mathrm{M}$ to $0.09 \mu \mathrm{M}$ by 3-fold dilution were diluted in maintenance medium. BHK cells were seeded in 96-well plate at a density of 5,000 cells per well and allowed to recover for $24 \mathrm{~h}$. Culture medium was replaced by maintenance medium containing the compound to be examined or drug-free. After 8 days' exposure, cell viability was also determined with Cell Titer-Glo reagent. The $\mathrm{CC}_{50}$ values were calculated by Origin 8.0.

\section{Funding Sources}

This work was financially supported by NSFC (81502923, 81773631, 81302702), National S\&T Major Special Project on Major New Drug Innovations (2018ZX09711001-007-002).

\section{Acknowledgement}

Thanks to Dr. Luke A. Kassekert (University of Minnesota) for help in the preparation of this manuscript.

\section{Notes}

The authors declare no competing financial interest. 


\section{ABBREVIATIONS}

ZIKV, Zika virus; YFV, yellow fever virus; DENV, dengue virus; WNV, west nile virus; WHO, world health organization; SIN, Sinefungin; SAM, S-Adenosyl methionine; Mtases, Methyltransferases; SAH, S-adenosyl-1-homocysteine; NITD, Novartis Institute for Tropical Disease.

\section{REFERENCES}

[1] V. Koppolu, T. Shantha Raju, Zika virus outbreak: a review of neurological complications, diagnosis, and treatment options, J Neurovirol. (2018) DOI: 10.1007/s13365-018-0614-8. .

[2] M.M. Rajah, R.D. Pardy, S.A. Condotta, M.J. Richer, S.M. Sagan, Zika Virus: Emergence, Phylogenetics, Challenges, and Opportunities, ACS Infect. Dis. 2 (2016) 763-772.

[3] H. Xia, X. Xie, C. Shan, P.Y. Shi, Potential Mechanisms for Enhanced Zika Epidemic and Disease, ACS Infect. Dis. (2018) DOI: 10.1021/acsinfecdis.8b00004..

[4]Centers for Disease Control and Prevention, All Countries \& territories with active zika virus Transmission. 2016, https://stacks.cdc.gov/view/cdc/42499.

[5] L. Yuan, X.Y. Huang, Z.Y. Liu, F. Zhang, X.L. Zhu, J.Y. Yu, X. Ji, Y.P. Xu, G. Li, C. Li, H.J. Wang, Y.Q. Deng, M. Wu, M.L. Cheng, Q. Ye, D.Y. Xie, X.F. Li, X. Wang, W. Shi, B. Hu, P.Y. Shi, Z. Xu, C.F. Qin, A single mutation in the prM protein of Zika virus contributes to fetal microcephaly, Science 358 (2017) 933-936.

[6] World Health Organization, The history of Zika virus, http://www.who.int/emergencies/zika-virus/ history/en/.

[7] R.L. Hamil, M.M. Hoehn, A9145, a new adenine-containing antifungal antibiotic. I. Discovery and isolation, J. Antibiot. 26 (1973) 463-465.

[8] R.S. Gordee, T.F. Butler, A9145, a new adenine-containing antifungal antibiotic. II. Biological activity, J. Antibiot. 26 (1973) 466-470.

[9] J.M. Zingg, J.C. Shen, A.S. Yang, H. Rapoport, P.A. Jones, Methylation inhibitors can increase the rate of cytosine deamination by (cytosine-5)-DNA methyltransferase, Nucleic Acids Res. 24 (1996) 3267-3275.

[10] H. Chen, B. Zhou, M. Brecher, N. Banavali, S.A. Jones, Z. Li, J. Zhang, D. Nag, L.D. Kramer, A.K. Ghosh, H. Li, S-adenosyl-homocysteine is a weakly bound inhibitor for a flaviviral methyltransferase, PloS one 8 (2013) e76900.

[11] C.S. Pugh, R.T. Borchardt, Effects of S-adenosylhomocysteine analogues on vaccinia viral messenger ribonucleic acid synthesis and methylation, Biochem. 21 (1982) 1535-1541.

[12] D.K. Dube, G. Mpimbaza, A.C. Allison, E. Lederer, L. Rovis, Antitrypanosomal activity of sinefungin, Am. J. Trop. Med. Hyg. 32 (1983) 31-33.

[13] B. Wang, S. Thurmond, R. Hai, J. Song, Structure and function of Zika virus NS5 protein: perspectives for drug design, Cell. Mol. life Sci. 2018, DOI: 10.1007/s00018-018-2751-x.

[14] K. Hercik, J. Brynda, R. Nencka, E. Boura, Structural basis of Zika virus methyltransferase inhibition by sinefungin, Arch. Virol. 162 (2017) 2091-2096.

[15] R.P.M. Abrams, J. Solis, A. Nath, Therapeutic Approaches for Zika Virus Infection of the Nervous System, Neurotherapeutics, 14 (2017) 1027-1048.

[16] C.A. Lipinski, F. Lombardo, B.W. Dominy, P.J. Feeney, Experimental and computational approaches to estimate solubility and permeability in drug discovery and development settings, Adv. Drug Deliv. Rev. 46 (2001) 3-26. 
[17] S.R. Daigle, E.J. Olhava, C.A. Therkelsen, C.R. Majer, C.J. Sneeringer, J. Song, L.D. Johnston, M.P. Scott, J.J. Smith, Y. Xiao, L. Jin, K.W. Kuntz, R. Chesworth, M.P. Moyer, K.M. Bernt, J.C. Tseng, A.L. Kung, S.A. Armstrong, R.A. Copeland, V.M. Richon, R.M. Pollock, Selective killing of mixed lineage leukemia cells by a potent small-molecule DOT1L inhibitor, Cancer cell 20 (2011) 53-65.

[18] S.P. Lim, L.S. Sonntag, C. Noble, S.H. Nilar, R.H. Ng, G. Zou, P. Monaghan, K.Y. Chung, H. Dong, B. Liu, C. Bodenreider, G. Lee, M. Ding, W.L. Chan, G. Wang, Y.L. Jian, A.T. Chao, J. Lescar, Z. Yin, T.R. Vedananda, T.H. Keller, P.Y. Shi, Small molecule inhibitors that selectively block dengue virus methyltransferase, J. Bio. Chem. 286 (2011) 6233-6240. 


\section{Highlights:}

1. $\mathrm{SIN}\left(\mathrm{IC}_{50}>50 \mu \mathrm{m}\right)$ proved to be inactive against ZIKV.

2. 1ad-af, 1ba-bb, and 1bf-bh displays potent anti-ZIKV activity $\left(\mathrm{IC}_{50}=4.56-20.16 \mu \mathrm{M}\right)$.

3. 9a exhibits good activity $\left(\mathrm{IC}_{50}=29.98 \mu \mathrm{M}\right)$ and acceptable cytotoxicity $\left(\mathrm{CC}_{50}>200 \mu \mathrm{M}\right)$. 